\title{
Catalytic Asymmetric Transfer Hydrogenation of $\alpha$-Ketoesters with Hantzsch Esters
}

\author{
Jung Woon Yang* and Benjamin List* \\ Max-Planck-Institut für Kohlenforschung, Kaiser-Wilhelm-Platz 1, 45470 \\ Mülheim an der Ruhr, Germany.
}

E-mail: list@mpi-muelheim.mpg.de; jwyang@mpi-muelheim.mpg.de

General: Reactions were monitored by thin layer chromatography using $0.25 \mathrm{~mm}$ E. Merck silica gel coated glass plates (60F-254) using UV light to visualize the course of reaction. Flash column chromatography was performed using E. Merck siliga gel (60, particle size 0.040-0.063 mm). Chemical yields refer to pure isolated substances. ${ }^{1} \mathrm{H}$ and ${ }^{13} \mathrm{C}$ NMR spectra were obtained using either a Bruker DPX-300 or AV-400 spectrometer. Chemical shifts are reported in ppm from tetramethylsilane with the solvent resonance as the internal standard. The following abbreviations were used to designate chemical shift mutiplicities: $\mathrm{s}=$ singlet, $\mathrm{d}$ = doublet, $\mathrm{t}=$ triplet, $\mathrm{q}=$ quartet, $\mathrm{h}=$ heptet, $\mathrm{m}=$ multiplet, $\mathrm{br}=$ broad. $\mathrm{MS}$ (EI): Finnigan MAT 8200 (70 eV), ESI-MS: Finnigan MAT 95, accurate mass determinations: Bruker APEX III FT-MS (7 T magnet).

\section{Typical procedure for the preparation of Hantzsch ester [4b]: ${ }^{1}$}

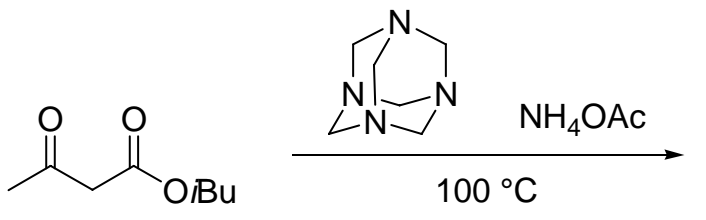<smiles>CCCCOC(=O)C1=C(C)NC(C)=C(C(=O)OCCC)[CH]1</smiles>

$4 \mathbf{b}$

A round-bottomed flask was charged with a mixture of neopentyl 3-oxobutanoate (14.00 g, $81.29 \mathrm{mmol}$ ), ammonium acetate (3.16 g, $40.65 \mathrm{mmol}$ ), hexamethylenetetramine (5.70 g, $40.65 \mathrm{mmol}$ ). The reaction mixture was heated to $100^{\circ} \mathrm{C}$ with stirring for $20 \mathrm{~min}$, and then cooled to room temperature. The reaction mixture was quenched with $\mathrm{H}_{2} \mathrm{O}$ and extracted with dichloromethane. The combined organic layer were dried over $\mathrm{MgSO}_{4}$, filtered and concentrated in vacuo. The residue was purified by flash column chromatography on silica gel

(1) Torchy1, S.; Cordonnier1, G.; Barbry1, D.; Eynde, J. J. V. Molecules 2002, 7, 528-533. 
(10\% ethyl acetate in hexane) to give Hantzsch ester $\mathbf{4 b}$ (8.91 g, 65\% yield) as pale yellowish solid.

${ }^{1} \mathrm{H}$ NMR (300 MHz, $\mathrm{CDCl}_{3}$ ) $\delta 5.23$ (brs, 1H), 3.70 (s, 4H), 3.31(s, 2H), 2.14 (s, 6H), 0.89 (s, $18 \mathrm{H}) ;{ }^{13} \mathrm{C}$ NMR (75.5 MHz, $\left.\mathrm{CDCl}_{3}\right) \delta$ 168.3, 145.4, 100.0, 73.6, 31.7, 26.9, 25.3, 19.3; HRMS (ESI) calcd for $\mathrm{MNa}^{+} 360.21453$, found 360.21448.

Typical procedure for the preparation of aliphatic substituted $\alpha$-ketoester [t-butyl $t$ butylglyoxylate]: ${ }^{2}$<smiles>CC(C)(C)C(=O)C(=O)O</smiles>

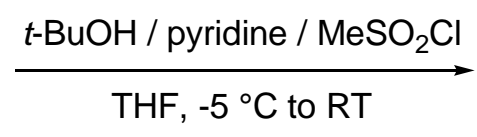<smiles>CCCOC(=O)C(=O)C(C)(C)C</smiles>

11

Trimethylpyruvic acid (5.00 g, $38.42 \mathrm{mmol})^{3}$ was dissolved in a mixture of THF (50 $\mathrm{mL}), t-\mathrm{BuOH}(5.70 \mathrm{~g}, 76.84 \mathrm{mmol})$ and pyridine $(7.60 \mathrm{~g}, 0.978 \mathrm{mmol})$. The mixture was cooled with stirring to $-5{ }^{\circ} \mathrm{C}$. $\mathrm{MeSO}_{2} \mathrm{Cl}(5.28 \mathrm{~g}, 46.10 \mathrm{mmol})$ was added dropwise over $5 \mathrm{~min}$. Precipitation of pyridinium salt began immediately. The suspension was stirred at room temperature overnight. The reaction mixture was quenched with $\mathrm{H}_{2} \mathrm{O}$ and extracted with diethyl ether. The combined organic layer were dried over $\mathrm{MgSO}_{4}$, filtered and concentrated in vacuo. The residual oil was distilled under vacuum to afford corresponding product as colorless oil in $72 \%$ yield (5.14 g).

Typical procedure for the preparation of aromatic substituted $\alpha$-ketoester [t-butyl 2naphthoyl formate]: ${ }^{4}$<smiles>CCCCC(=O)C(=O)n1ccnc1</smiles>

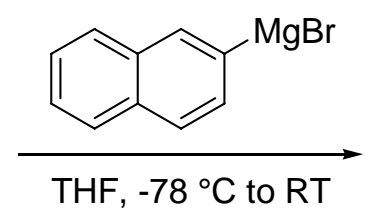<smiles>CCOC(=O)C(=O)c1ccc2ccccc2c1</smiles>

1i

The $t$-butyl $\alpha$-oxo-1H-imidazole-1-acetate (4.90 g, $24.97 \mathrm{mmol}$ ) was dissolved in THF (100 mL) under argon and cooled to $-78{ }^{\circ} \mathrm{C}$ in a dry ice/acetone bath. The 2naphthylmagnesium bromide solution (0.5 M in THF, $24.97 \mathrm{mmol}$ ) was added by dropping

(2) Hegarty, A. F.; O’Neill, P. Synthesis 1993, 606-610.

(3) Koch, C.-J.; Šimonyiová, S.; Pabel, J.; Kärtner, A.; Polborn, K.; Wanner, K. T. Eur. J. Org. Chem. 2003, 1244-1263.

(4) Nimitz, J. S.; Mosher, H. S. J. Org. Chem. 1981, 46, 211-213. 
funnel over 15 min with stirring, and then the reaction mixture was warmed to room temperature. The reaction mixture was quenched with saturated $\mathrm{NH}_{4} \mathrm{Cl}$ solution and extracted with diethyl ether. The combined organic layer were dried over $\mathrm{MgSO}_{4}$, filtered and concentrated in vacuo. The residue was purified by flash column chromatography on silica gel (5\% ethyl acetate in hexane) to give $2.88 \mathrm{~g}$ ( $45 \%$ yield) of corresponding product.<smiles>CCOC(=O)C(=O)c1ccccc1</smiles>

${ }^{1} \mathrm{H}$ NMR (300 MHz, $\left.\mathrm{CDCl}_{3}\right) \delta 7.90(\mathrm{~d}, J=8.1 \mathrm{~Hz}, 2 \mathrm{H}), 7.55$ (t, $J=7.4 \mathrm{~Hz}$, 1H), 7.43 (t, $J=6.6 \mathrm{~Hz}, 2 \mathrm{H}), 1.60$ (s, 9H); $\left.{ }^{13} \mathrm{C} \mathrm{NMR} \mathrm{(75.5} \mathrm{MHz,} \mathrm{CDCl}_{3}\right) \delta 187.2,164.1$, 135.0, 132.9, 130.2, 129.2, 85.1, 28.4; HRMS (CI, isobutane) calcd for $\mathrm{MH}^{+}$207.10157, found 207.10166.<smiles>CCCOC(=O)C(=O)c1ccc([N+](=O)[O-])cc1</smiles>

${ }^{1} \mathrm{H}$ NMR (300 MHz, $\left.\mathrm{CDCl}_{3}\right) \delta 8.28(\mathrm{~d}, J=8.9 \mathrm{~Hz}, 2 \mathrm{H}), 8.11(\mathrm{~d}, J=$ 8.9 Hz, 2H), 1.57(s, 9H); ${ }^{13} \mathrm{C}$ NMR (75.5 MHz, $\left.\mathrm{CDCl}_{3}\right) \delta 185.1,162.5,151.4,137.5,131.4$, 124.3, 86.2, 28.4; HRMS (CI, isobutane) calcd for $\mathrm{MH}^{+}$252.08695, found 252.08719.<smiles>CCOC(=O)C(=O)c1ccc(Cl)cc1</smiles>

${ }^{1} \mathrm{H}$ NMR (300 MHz, $\mathrm{CDCl}_{3}$ ) $\delta 7.84$ (d, $\left.J=8.7 \mathrm{~Hz}, 2 \mathrm{H}\right), 7.38$ (d, $J=8.7$ $\mathrm{Hz}, 2 \mathrm{H}), 1.53(\mathrm{~s}, 9 \mathrm{H}) ;{ }^{13} \mathrm{C} \mathrm{NMR}\left(75.5 \mathrm{MHz}, \mathrm{CDCl}_{3}\right) \delta 185.7,163.4,141.6,131.5,131.3,129.9$, 85.4, 28.4; HRMS (ESI) calcd for $\mathrm{MNa}^{+}$263.04448, found 263.04454.<smiles>CCCOC(=O)C(=O)c1ccc(C)cc1</smiles>

${ }^{1} \mathrm{H}$ NMR (300 MHz, $\left.\mathrm{CDCl}_{3}\right) \delta 7.86(\mathrm{~d}, J=8.2 \mathrm{~Hz}, 2 \mathrm{H}), 7.30$ (d, $J=8.0$ $\mathrm{Hz}, 2 \mathrm{H}), 2.43(\mathrm{~s}, 3 \mathrm{H}), 1.62$ (s, 9H); ${ }^{13} \mathrm{C} \mathrm{NMR}\left(75.5 \mathrm{MHz}, \mathrm{CDCl}_{3}\right) \delta 186.5,163.9,145.9,130.1$, 130.0, 129.6, 84.5, 28.1, 21.9; HRMS (ESI) calcd for $\mathrm{MNa}^{+}$243.09936, found 243.09916. 
<smiles>CCCOC(=O)C(=O)c1ccc(OC)cc1</smiles>

${ }^{1} \mathrm{H}$ NMR (300 MHz, $\left.\mathrm{CDCl}_{3}\right) \delta 7.86(\mathrm{~d}, J=8.8 \mathrm{~Hz}, 2 \mathrm{H}), 6.88(\mathrm{~d}, J=$ $8.8 \mathrm{~Hz}, 2 \mathrm{H}), 3.80(\mathrm{~s}, 3 \mathrm{H}), 1.54$ (s, 9H); ${ }^{13} \mathrm{C} \mathrm{NMR}\left(75.5 \mathrm{MHz}, \mathrm{CDCl}_{3}\right) \delta 185.8,165.1,164.4$, 132.7, 125.9, 114.1, 84.8, 56.0, 28.4; HRMS (ESI) calcd for $\mathrm{MNa}^{+}$259.09388, found 259.09407.<smiles>CCOC(=O)C(=O)c1ccc2ccccc2c1</smiles>

${ }^{1} \mathrm{H}$ NMR (300 MHz, $\left.\mathrm{CDCl}_{3}\right) \delta 8.41$ (s, 1H), 7.95-7.78 (m, 4H), 7.587.45 (m, 2H), 1.59 (s, 9H); ${ }^{13} \mathrm{C}$ NMR (75.5 MHz, $\left.\mathrm{CDCl}_{3}\right) \delta$ 187.2, 164.2, 136.6, 133.5, 132.7, 130.3, 130.2, 129.8, 129.3, 128.3, 127.5, 124.4, 85.2, 28.5; HRMS (ESI) calcd for $\mathrm{MNa}^{+}$ 279.09919, found 279.09916.<smiles>CCCOC(=O)C(=O)c1ccco1</smiles>
6.62 (m, 1H), 1.62 (s, 9H); ${ }^{13} \mathrm{C}$ NMR (75.5 MHz, $\left.\mathrm{CDCl}_{3}\right) \delta$ 172.5, 160.6, 149.8, 149.2, 124.0, 112.9, 84.7, 27.9; HRMS (CI, isobutane) calcd for $\mathrm{MH}^{+}$197.08125, found 197.08138.<smiles>CCOC(=O)C(=O)C1CCCCC1</smiles>

${ }^{1} \mathrm{H}$ NMR (300 MHz, $\left.\mathrm{CDCl}_{3}\right) \delta 2.95$ (brs, 1H), 1.90-1.70 (m, 5H), 1.55 (s, 9H), 1.37-1.23 (m, 5H); ${ }^{13} \mathrm{C}$ NMR (75.5 MHz, $\left.\mathrm{CDCl}_{3}\right) \delta$ 198.5, 161.8, 83.8, 46.1, 27.9, 27.7, 25.5, 25.3; HRMS (CI, isobutane) calcd for $\mathrm{MH}^{+}$213.14852, found 213.14881.<smiles>CCCOC(=O)C(=O)C(C)(C)C</smiles>

1I NMR spectra are in accordance with the literature. ${ }^{4}$ 
Table. Influence of the Hantzsch Ester

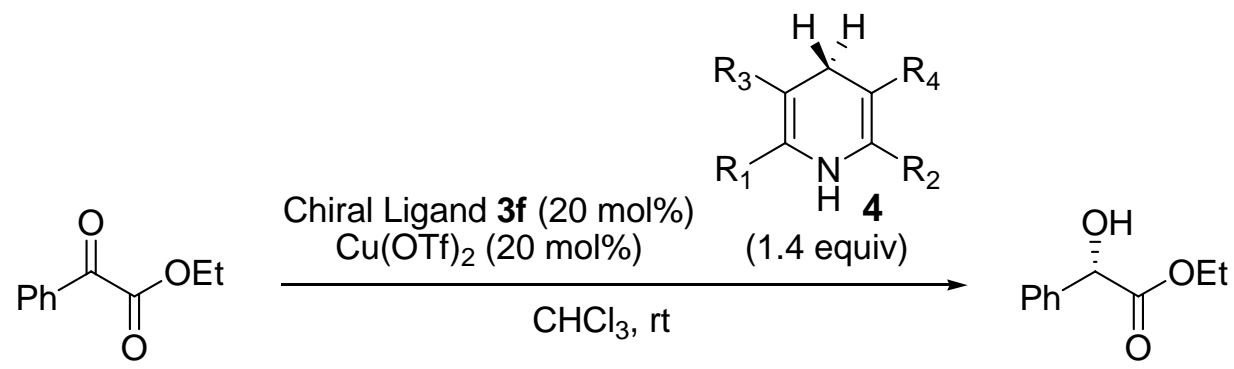

$1 \mathrm{a}$

$(S)-2 a$

\begin{tabular}{ccccccccc}
\hline entry & $\begin{array}{c}\text { Hantzsch } \\
\text { ester }\end{array}$ & $\mathrm{R}_{1}$ & $\mathrm{R}_{2}$ & $\mathrm{R}_{3}$ & $\mathrm{R}_{4}$ & $\begin{array}{c}\text { time } \\
(\mathrm{h})\end{array}$ & $\begin{array}{c}\text { yield } \\
(\%)^{a}\end{array}$ & $e^{b}$ \\
\hline 1 & $\mathbf{4 a}$ & $\mathrm{Me}$ & $\mathrm{Me}$ & $\mathrm{CO}_{2} \mathrm{Et}$ & $\mathrm{CO}_{2} \mathrm{Et}$ & 7 & 90 & $91: 9$ \\
$\mathbf{2}$ & $\mathbf{4 b}$ & $\mathbf{M e}$ & $\mathbf{M e}$ & $\mathbf{C O}_{2} \mathbf{i B u}$ & $\mathbf{C O}_{2} \mathbf{i B u}$ & $\mathbf{4}$ & $\mathbf{9 7}$ & $\mathbf{9 1 : 9}$ \\
3 & $\mathbf{4 c}$ & $\mathrm{Pr}$ & $\mathrm{Pr}$ & $\mathrm{CO}_{2} \mathrm{Me}$ & $\mathrm{CO}_{2} \mathrm{Me}$ & 4 & 90 & $90: 10$ \\
4 & $\mathbf{4 d}$ & $\mathrm{Me}$ & $\mathrm{Me}$ & $\mathrm{CO}_{2} \mathrm{Me}$ & $\mathrm{CO}_{2} t \mathrm{Bu}$ & 5 & 85 & $92: 8$ \\
5 & $\mathbf{4 e}$ & $\mathrm{Me}$ & $i \mathrm{Pr}$ & $\mathrm{CO}_{2} \mathrm{Me}$ & $\mathrm{CO}_{2} \mathrm{Me}$ & 48 & $<5$ & n.d
\end{tabular}

${ }^{a}$ Isolated yield. ${ }^{b}$ Determined by chiral HPLC analysis on Chiralcel OD-H column.

\section{General procedure for $\mathrm{Cu}(\mathrm{II})$-Bisoxazoline-Catalyzed Asymmetric Transfer Hydrogenation of $\alpha$-Ketoesters with Hantzsch Esters.}

To a flame-dried Schlenk tube was added $\mathrm{Cu}(\mathrm{OTf})_{2}(18.1 \mathrm{mg}, 0.05 \mathrm{mmol})$ and $[(S, S)-$ benzyl-box] 3f (18.1 mg, $0.05 \mathrm{mmol})$. The mixture was dried under vacuum for $0.5 \mathrm{~h}$ and distilled anhydrous $\mathrm{CHCl}_{3}(2.5 \mathrm{~mL})$ was added. After stirring for $1 \mathrm{~h}, t$-butyl benzoylformate 1d (103.1 mg, $0.5 \mathrm{mmol}$ ) was added and the mixture was cooled to $-25{ }^{\circ} \mathrm{C}$. After addition of Hantzsch ester $\mathbf{4 b}$ (216.6 $\mathrm{mg}, 0.7 \mathrm{mmol}$ ), the mixture was stirred under argon at the same temperature until disappearance of the starting material (48 h). After conclusion of the reaction, the mixture was washed using $\mathrm{H}_{2} \mathrm{O}$ and the aqueous layer was extracted with $\mathrm{CHCl}_{3}$. The organic layer was dried $\left(\mathrm{MgSO}_{4}\right)$, filtered, and concentrated in vacuo to yield the crude product as an off-white solid. Purification by column chromatography (15\% EtOAc/hexanes) gave the pure product as a white solid (92.2 $\mathrm{mg}, 89 \%)$, identical in all respects to previously described $t$-butyl (S)-mandelate 2d. $[\alpha]_{\mathrm{D}}+90.7$ (c 1.0, MeOH); $\left[[\alpha]_{\mathrm{D}}+97.4\right.$ (c 1.0, $\left.\mathrm{MeOH}\right)$, for $>99: 1$ e.r.]. ${ }^{5}$

(5) Ebbers, E. J.; Ariaans, G. J. A.; Bruggink, A.; Zwanenburg, B. Tetrahedron: Asymmetry 1999, 10, 3701-3718. 
<smiles>CCCCOC(=O)[C@@H](O)c1ccccc1</smiles>

${ }^{1} \mathrm{H}$ NMR (300 MHz, $\left.\mathrm{CDCl}_{3}\right) \delta$ 7.35-7.24 (m, 5H), 4.96 (d, $\left.J=5.9 \mathrm{~Hz}, 1 \mathrm{H}\right)$, 3.49 (d, $J=5.9 \mathrm{~Hz}, 1 \mathrm{H}), 1.32$ (s, 9H); ${ }^{13} \mathrm{C} \mathrm{NMR}\left(75.5 \mathrm{MHz}, \mathrm{CDCl}_{3}\right) \delta 173.3,139.3,128.6$, 128.5, 126.8, 83.4, 73.4, 28.2; HRMS (CI, isobutane) calcd for $\mathrm{MH}^{+}$209.11777, found 209.11782; The enantiomeric ratio was determined to be 96:4 by chiral HPLC (Chiralcel OD-H column, 3\% i-PrOH/heptane, $1.0 \mathrm{~mL} / \mathrm{min}, 220 \mathrm{~nm}, \mathrm{t}_{\mathrm{R}}$ (major $7.4 \mathrm{~min}$ ), $\mathrm{t}_{\mathrm{R}}$ (minor $18.0 \mathrm{~min}$ ).<smiles>CCCOC(=O)[C@H](O)c1ccc([N+](=O)[O-])cc1</smiles>

${ }^{1} \mathrm{H}$ NMR (300 MHz, $\left.\mathrm{CDCl}_{3}\right) \delta 8.14$ (d, $\left.J=8.7 \mathrm{~Hz}, 2 \mathrm{H}\right), 7.56$ (d, $J=$ $8.7 \mathrm{~Hz}, 2 \mathrm{H}), 5.09$ (d, $J=5.1 \mathrm{~Hz}, 1 \mathrm{H}), 3.69$ (d, $J=5.1 \mathrm{~Hz}, 1 \mathrm{H}) 1.34$ (s, 9H); ${ }^{13} \mathrm{C}$ NMR $(75.5$ $\left.\mathrm{MHz}, \mathrm{CDCl}_{3}\right) \delta 171.9,148.1,146.3,127.6,123.9,84.6,72.6,28.1$; HRMS (CI, isobutane) calcd for $\mathrm{MH}^{+}$254.10290, found 254.10284; The enantiomeric ratio was determined to be 90:10 by chiral HPLC (Chiralcel OD-H column, 2\% $\mathrm{i}$-PrOH/heptane, $1.0 \mathrm{~mL} / \mathrm{min}, 220 \mathrm{~nm}, \mathrm{t}_{\mathrm{R}}$ (major $16.8 \mathrm{~min}), \mathrm{t}_{\mathrm{R}}$ (minor $\left.18.4 \mathrm{~min}\right)$.<smiles>CCCOC(=O)[C@@H](O)c1ccc(Cl)cc1</smiles>

${ }^{1} \mathrm{H}$ NMR (300 MHz, $\left.\mathrm{CDCl}_{3}\right) \delta$ 7.29-7.22 (m, 4H), 4.94 (d, $J=5.0 \mathrm{~Hz}$, $1 \mathrm{H}), 3.51$ (d, $J=5.4 \mathrm{~Hz}, 1 \mathrm{H}) 1.33$ (s, 9H); $\left.{ }^{13} \mathrm{C} \mathrm{NMR} \mathrm{(75.5} \mathrm{MHz,} \mathrm{CDCl}_{3}\right) \delta 172.8,137.8$, 134.3, 128.9, 128.1, 83.8, 72.7, 28.2; HRMS (ESI) calcd for $\mathrm{MNa}^{+}$265.06009, found 265.06019; The enantiomeric ratio was determined to be 94:6 by chiral HPLC (Chiralcel OD$\mathrm{H}$ column, 3\% $\mathrm{i}$-PrOH/heptane, $1.0 \mathrm{~mL} / \mathrm{min}, 220 \mathrm{~nm}, \mathrm{t}_{\mathrm{R}}$ (major $\left.13.1 \mathrm{~min}\right), \mathrm{t}_{\mathrm{R}}$ (minor $14.1 \mathrm{~min}$ ).<smiles>CCCCC(=O)C(O)c1ccc(C)cc1</smiles>

${ }^{1} \mathrm{H}$ NMR (300 MHz, $\left.\mathrm{CDCl}_{3}\right) \delta 7.20$ (d, $\left.J=8.0 \mathrm{~Hz}, 2 \mathrm{H}\right), 7.07$ (d, $J=8.0$ Hz, 2H), 4.92 (s, 1H), 3.35 (brs, 1H), 2.26 (s, 3H), 1.33 (s, 9H); ${ }^{13} \mathrm{C}$ NMR (75.5 MHz, $\mathrm{CDCl}_{3}$ ) $\delta$ 173.4, 138.1, 136.4, 129.5, 126.6, 83.3, 73.2, 28.2, 21.5; HRMS (ESI) calcd for $\mathrm{MNa}^{+}$ 245.11482, found 245.11478; The enantiomeric ratio was determined to be 96:4 by chiral 
HPLC (Chiralcel OD-H column, 3\% i-PrOH/heptane, $1.0 \mathrm{~mL} / \mathrm{min}, 220 \mathrm{~nm}$, $\mathrm{t}_{\mathrm{R}}$ (major $6.8 \mathrm{~min}$ ), $\mathrm{t}_{\mathrm{R}}(\operatorname{minor} 13.3 \mathrm{~min})$.<smiles>CCCOC(=O)[C@H](O)c1ccc(OC)cc1</smiles>

${ }^{1} \mathrm{H}$ NMR $\left(300 \mathrm{MHz}, \mathrm{CDCl}_{3}\right) \delta 7.24(\mathrm{~d}, J=8.7 \mathrm{~Hz}, 2 \mathrm{H}), 6.80(\mathrm{~d}, J=$ $8.7 \mathrm{~Hz}, 2 \mathrm{H}), 4.91$ (d, $J=4.8 \mathrm{~Hz}, 1 \mathrm{H}), 3.73$ (s, 3H), 3.36 (d, $J=4.8 \mathrm{~Hz}, 1 \mathrm{H}), 1.33$ (s, 9H); ${ }^{13} \mathrm{C}$ NMR (75.5 MHz, $\mathrm{CDCl}_{3}$ ) $\delta 173.5,159.8,131.6,128.0$, 114.2, 83.3, 72.9, 55.6, 28.2; HRMS (ESI) calcd for $\mathrm{MNa}^{+}$261.10973, found 261.10984; The enantiomeric ratio was determined to be 97:3 by chiral HPLC (Chiralcel OD-H column, 3\% i-PrOH/heptane, $1.0 \mathrm{~mL} / \mathrm{min}, 220 \mathrm{~nm}$, $t_{R}\left(\right.$ major 9.4 min), $t_{R}($ minor $22.9 \mathrm{~min})$.<smiles>CCCOC(=O)[C@H](O)c1ccc2ccccc2c1</smiles>

${ }^{1} \mathrm{H}$ NMR (300 MHz, $\left.\mathrm{CDCl}_{3}\right) \delta 7.82$ (s, 1H), 7.82-7.74 (m, 3H), 7.457.39 (m, 3H), 5.13 (d, $J=5.6 \mathrm{~Hz}, 1 \mathrm{H}), 3.57$ (d, $J=5.7 \mathrm{~Hz}, 1 \mathrm{H}), 1.33$ (s, 9H); ${ }^{13} \mathrm{C}$ NMR $(75.5$ $\left.\mathrm{MHz}, \mathrm{CDCl}_{3}\right) \delta 173.2,136.7,133.6,133.5,128.6,128.5,128.0,126.6,126.5,126.0,124.5$, 83.6, 73.5, 28.2; HRMS (ESI) calcd for $\mathrm{MNa}^{+}$281.11481, found 281.11462; The enantiomeric ratio was determined to be 97:3 by chiral HPLC (Chiralcel OD-H column, 3\% i$\mathrm{PrOH} /$ heptane, $1.0 \mathrm{~mL} / \mathrm{min}, 220 \mathrm{~nm}, \mathrm{t}_{\mathrm{R}}$ (major $11.3 \mathrm{~min}$ ), $\mathrm{t}_{\mathrm{R}}$ (minor $13.9 \mathrm{~min}$ ).<smiles>CCCCOC(=O)[C@H](O)c1ccco1</smiles>

${ }^{1} \mathrm{H}$ NMR (300 MHz, $\left.\mathrm{CDCl}_{3}\right) \delta$ 7.39-7.38 (m, 1H), 6.36-6.32 (m, 2H), 5.05 (s, 1H), 3.38 (brs, 1H), 1.46 (s, 9H); ${ }^{13} \mathrm{C}$ NMR (75.5 MHz, $\left.\mathrm{CDCl}_{3}\right) \delta$ 170.6, 151.6, 142.7, 110.4, 108.1, 83.5, 67.1, 27.8; HRMS (CI, isobutane) calcd for $\mathrm{MH}^{+}$199.09696, found 199.09703; The enantiomeric ratio was determined to be 97:3 by chiral HPLC (Chiralcel OD-H column, 3\% i$\mathrm{PrOH} /$ heptane, $1.0 \mathrm{~mL} / \mathrm{min}, 220 \mathrm{~nm}$, $\mathrm{t}_{\mathrm{R}}$ (major $9.0 \mathrm{~min}$ ), $\mathrm{t}_{\mathrm{R}}$ (minor $10.2 \mathrm{~min}$ ). 
<smiles>CCCCOC(=O)[C@H](O)C1CCCCC1</smiles>

${ }^{1} \mathrm{H}$ NMR (300 MHz, $\mathrm{CDCl}_{3}$ ) $\delta 3.88$ (d, $J=2.3 \mathrm{~Hz}, 1 \mathrm{H}$ ), 2.62 (brs, $1 \mathrm{H}$ ), 1.78-1.76 (m, 2H), 1.67-1.64 (m, 3H), 1.49 (s, 9H), 1.45-1.44 (m, 1H), 1.99-1.12 (m, 5H); ${ }^{13} \mathrm{C}$ NMR (75.5 MHz, $\left.\mathrm{CDCl}_{3}\right) \delta 174.1,82.3,74.8,42.0,29.1,28.1$, 26.2, 26.1; HRMS (ESI) calcd for $\mathrm{MNa}^{+}$237.14600, found 237.14611; The enantiomeric ratio was determined to be 90:10 by chiral GC analysis [diethyl tert-butyl $\beta$-cyclodex, $60{ }^{\circ} \mathrm{C} / 1 \mathrm{~min}, 130{ }^{\circ} \mathrm{C} / 20 \mathrm{~min}, 220^{\circ} \mathrm{C} / 10$ min, and $320^{\circ} \mathrm{C} /$ isothermal, 0.6 bar, $\mathrm{t}_{\mathrm{R}}$ (major $63.4 \mathrm{~min}$ ), $\mathrm{t}_{\mathrm{R}}$ (minor $64.4 \mathrm{~min}$ )].<smiles>CCCCOC(=O)[C@H](O)C(C)(C)C</smiles>

2I $\quad{ }^{1} \mathrm{H}$ NMR (300 MHz, $\left.\mathrm{CDCl}_{3}\right) \delta 3.67$ (s, 1H), 2.84 (brs, 1H), 1.50 (s, 9H), 0.97 (s, 9H); ${ }^{13} \mathrm{C}$ NMR (75.5 MHz, $\left.\mathrm{CDCl}_{3}\right) \delta$ 173.7, 82.5, 78.4, 35.3, 28.1, 25.9; HRMS (CI, isobutane) calcd for $\mathrm{MH}^{+}$189.14911, found 189.14907; The enantiomeric ratio was determined to be 92:8 by chiral GC analysis [diethyl tert-butyl $\beta$-cyclodex, $60{ }^{\circ} \mathrm{C} / 1 \mathrm{~min}, 86^{\circ} \mathrm{C} / 6 \mathrm{~min}$, $220^{\circ} \mathrm{C} / 5 \mathrm{~min}$, and $320^{\circ} \mathrm{C} /$ isothermal, 0.6 bar, $\mathrm{t}_{\mathrm{R}}$ (major $24.7 \mathrm{~min}$ ), $\mathrm{t}_{\mathrm{R}}$ (minor $25.0 \mathrm{~min}$ )]. 


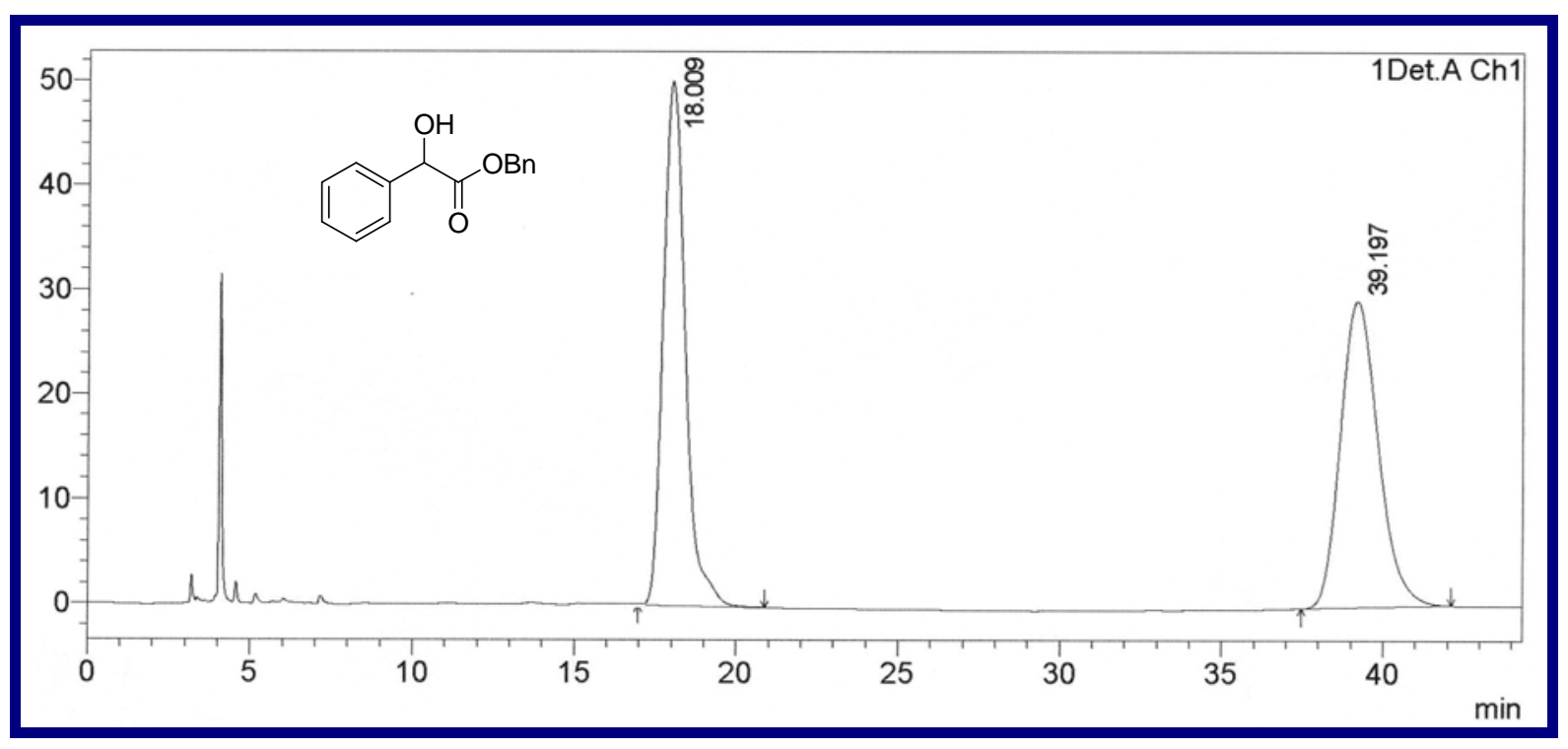

Detector A Ch1 220nm
\begin{tabular}{|l|r|r|}
\hline Peak \# & Ret. Time & Area $\%$ \\
\hline 1 & 18.009 & 49.859 \\
\hline 2 & 39.197 & 50.141 \\
\hline Total & & 100.000 \\
\hline
\end{tabular}

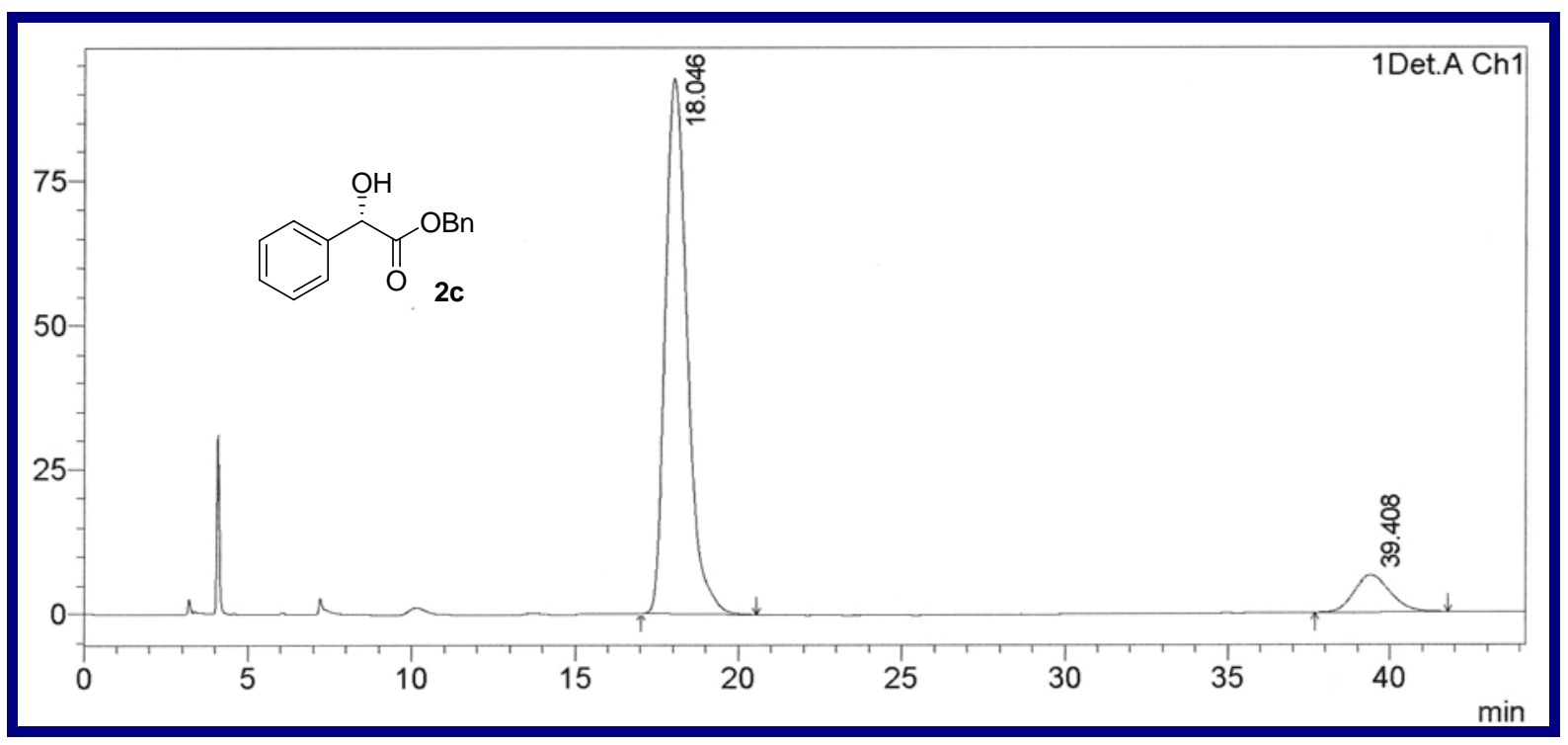

Detector A Ch1 220nm
\begin{tabular}{|l|r|r|}
\hline Peak \# & Ret. Time & Area $\%$ \\
\hline 1 & 18.046 & 89.632 \\
\hline 2 & 39.408 & 10.368 \\
\hline Total & & 100.000 \\
\hline
\end{tabular}




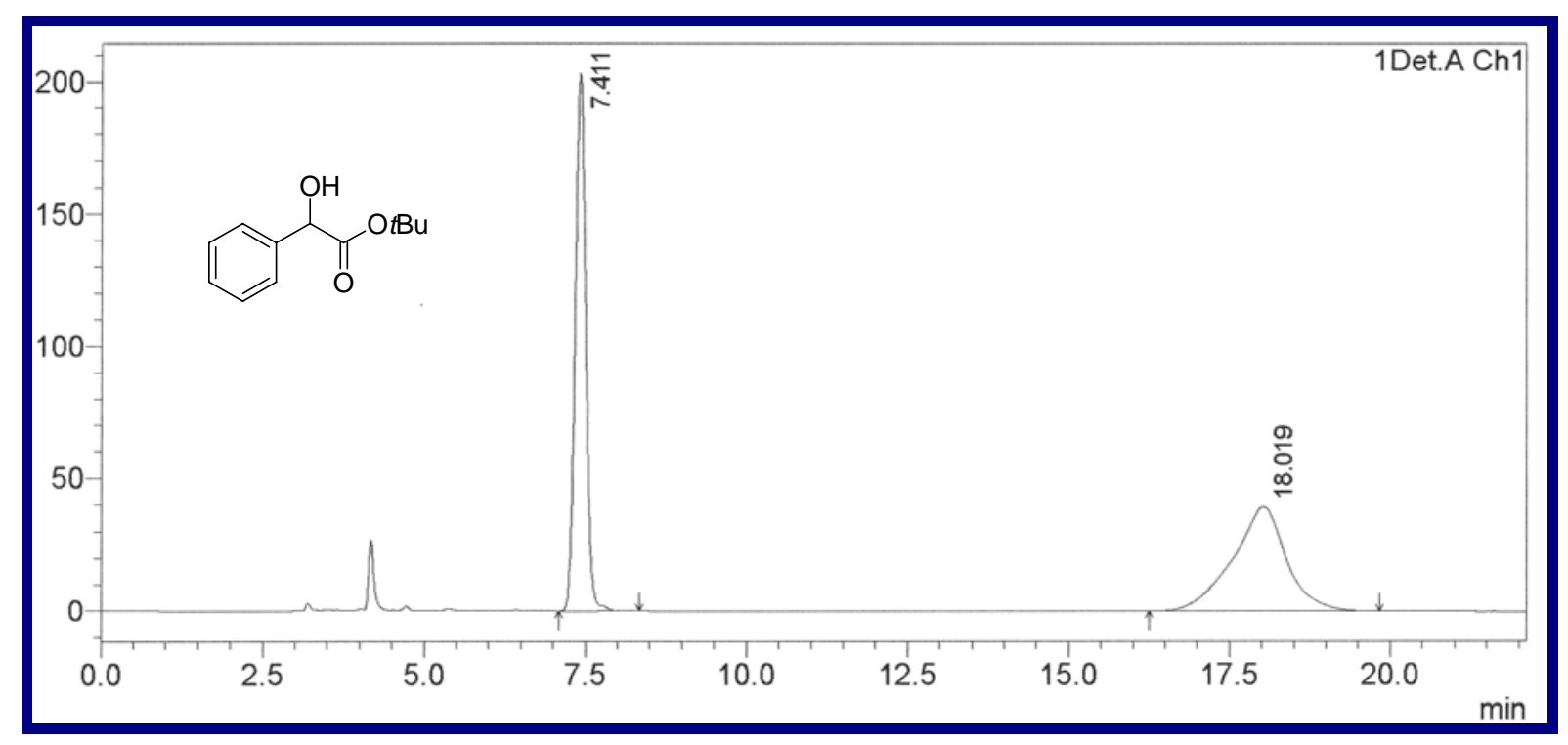

Detector A Ch1 220nm
\begin{tabular}{|l|r|r|}
\hline Peak\# & Ret. Time & \multicolumn{1}{|c|}{ Area $\%$} \\
\hline 1 & 7.411 & 50.034 \\
\hline 2 & 18.019 & 49.966 \\
\hline Total & & 100.000 \\
\hline
\end{tabular}

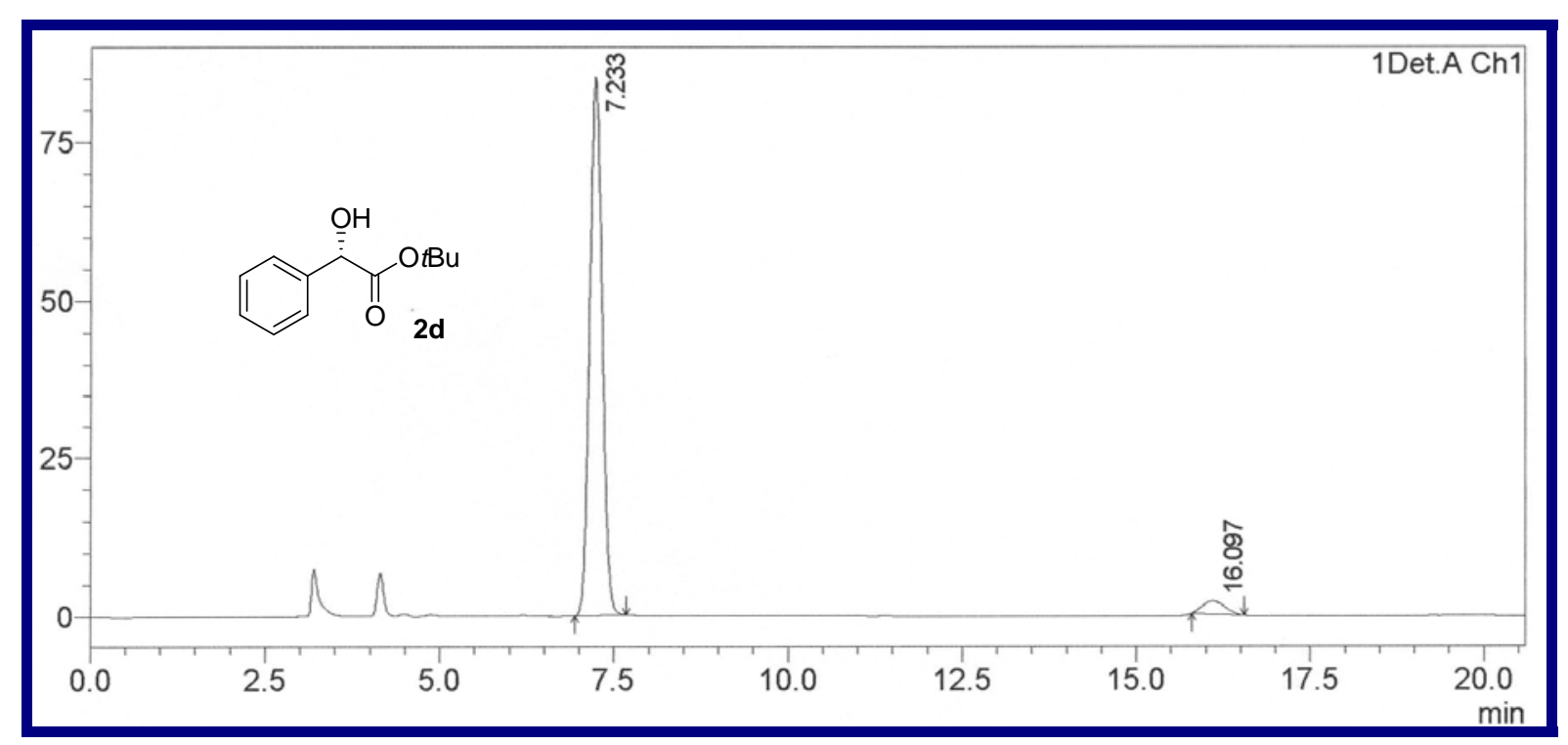

Detector A Ch1 220nm
\begin{tabular}{|l|r|r|}
\hline Peak \# & Ret. Time & Area $\%$ \\
\hline 1 & 7.233 & 95.963 \\
\hline 2 & 16.097 & 4.037 \\
\hline Total & & 100.000 \\
\hline
\end{tabular}




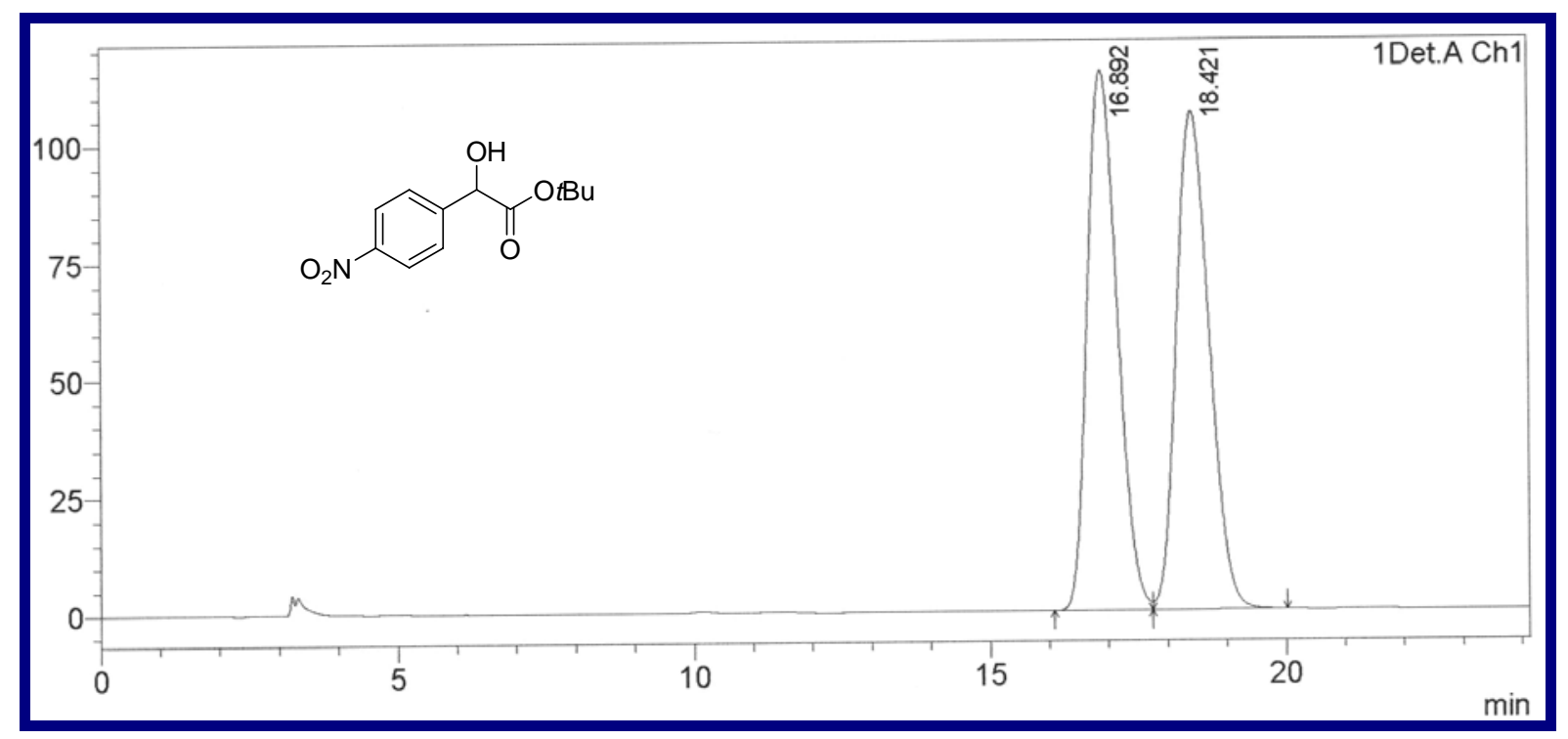

Detector A Ch1 220nm
\begin{tabular}{|l|r|r|}
\hline Peak \# & Ret. Time & Area $\%$ \\
\hline 1 & 16.892 & 49.877 \\
\hline 2 & 18.421 & 50.123 \\
\hline Total & & 100.000 \\
\hline
\end{tabular}

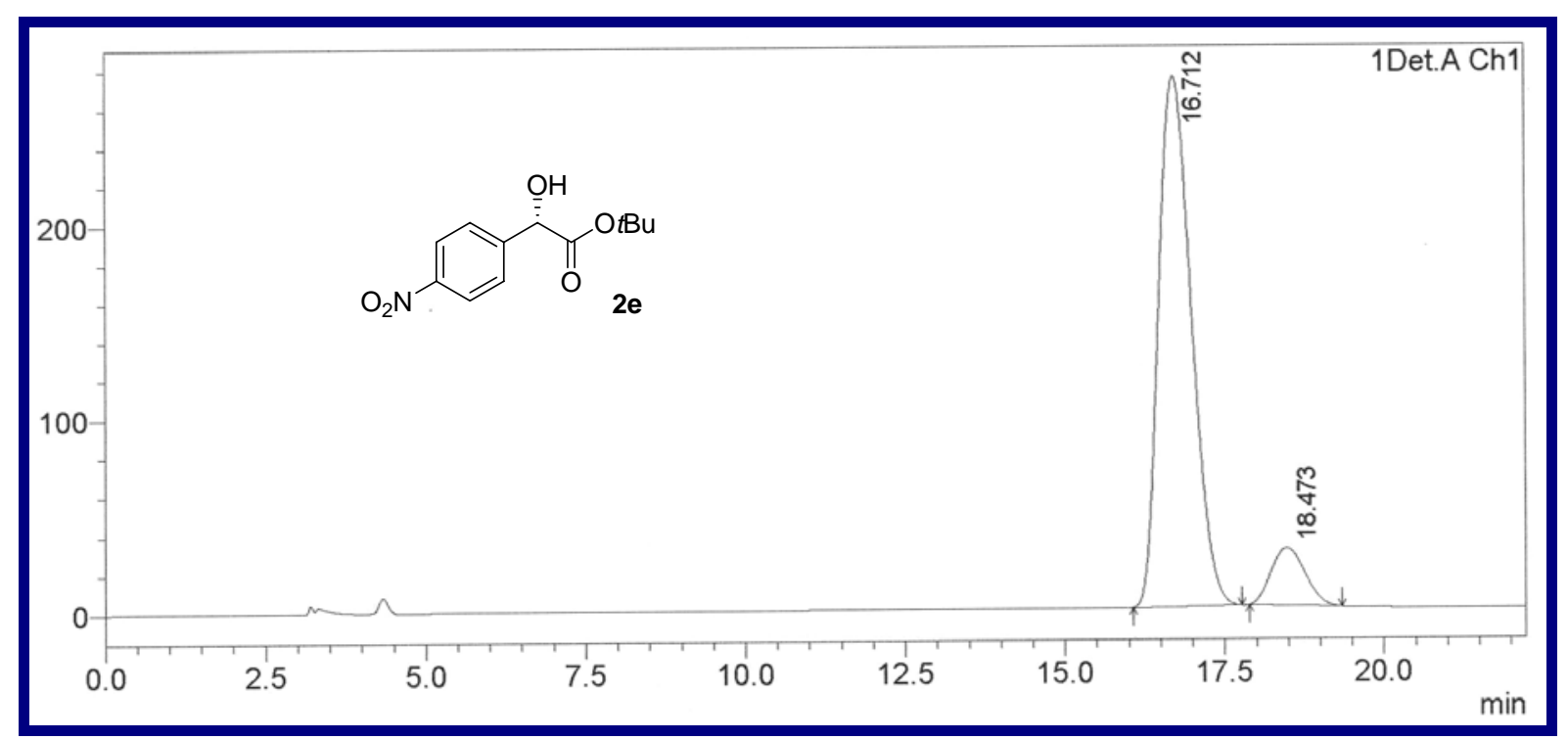

Detector A Ch1 220nm
\begin{tabular}{|l|r|r|}
\hline Peak \# & Ret. Time & Area $\%$ \\
\hline 1 & 16.712 & 89.898 \\
\hline 2 & 18.473 & 10.102 \\
\hline Total & & 100.000 \\
\hline
\end{tabular}




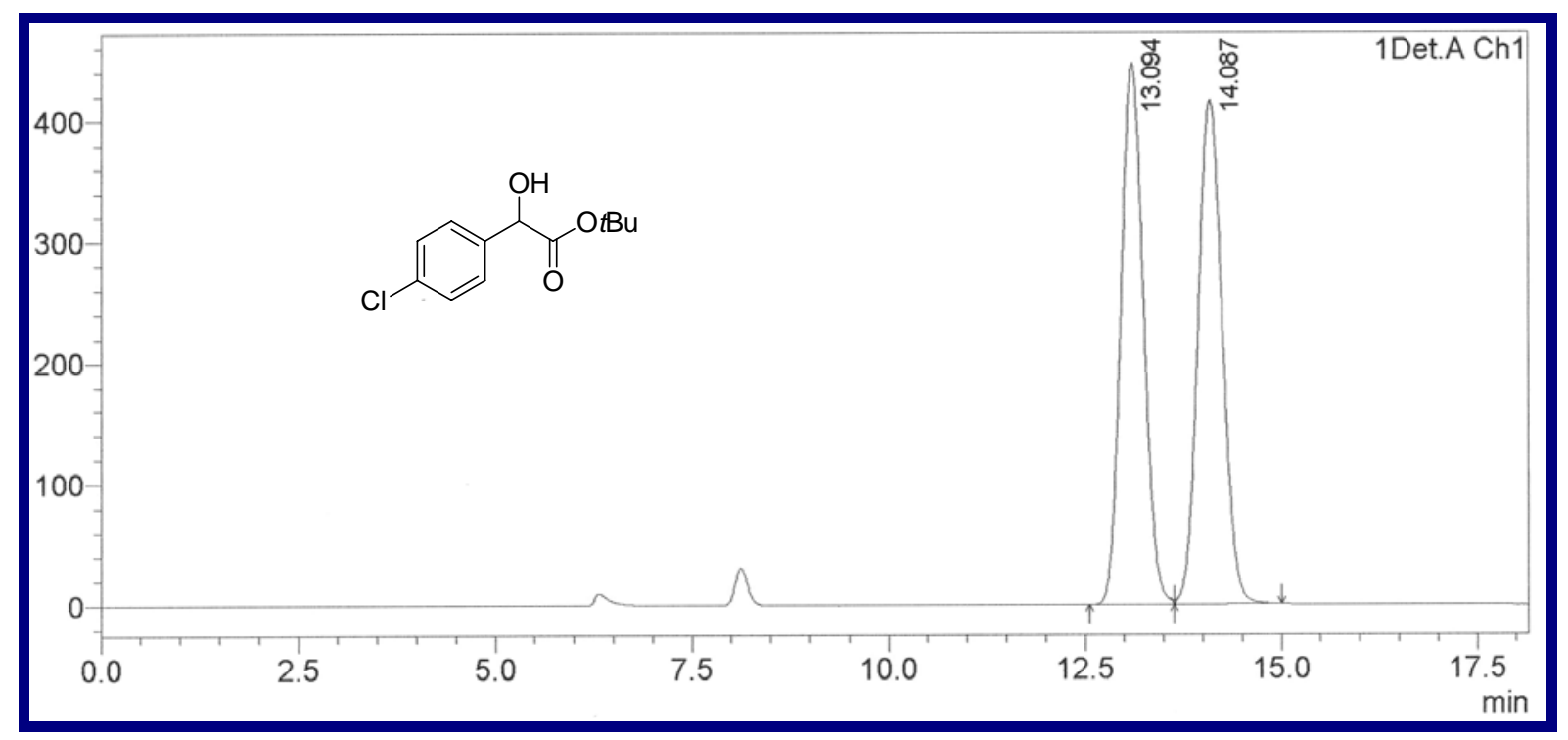

Detector A Ch1 220nm
\begin{tabular}{|l|r|r|}
\hline Peak \# & Ret. Time & Area $\%$ \\
\hline 1 & 13.094 & 49.945 \\
\hline 2 & 14.087 & 50.055 \\
\hline Total & & 100.000 \\
\hline
\end{tabular}

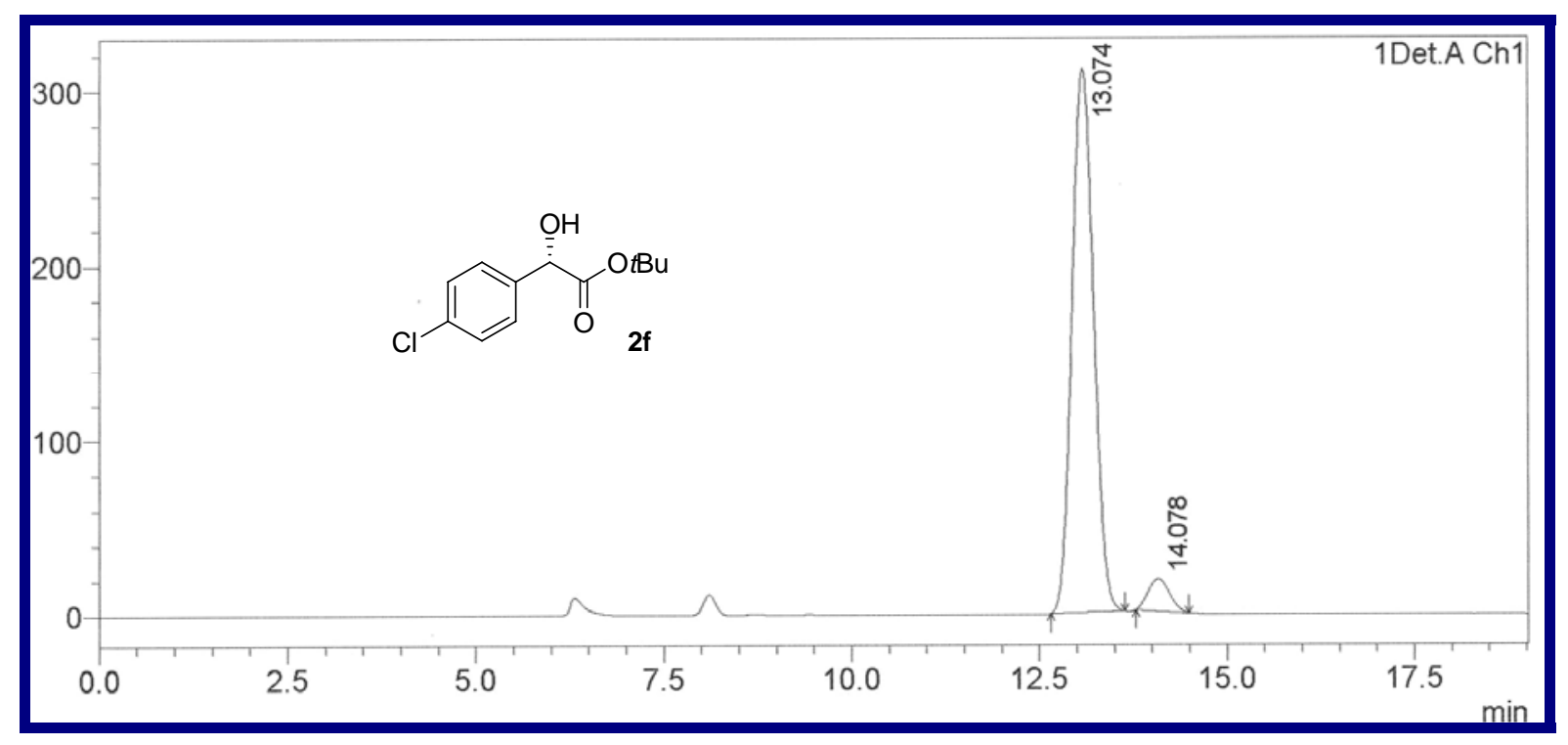

Detector A Ch1 220nm
\begin{tabular}{|l|r|r|}
\hline Peak\# & Ret. Time & Area $\%$ \\
\hline 1 & 13.074 & 94.449 \\
\hline 2 & 14.078 & 5.551 \\
\hline Total & & 100.000 \\
\hline
\end{tabular}




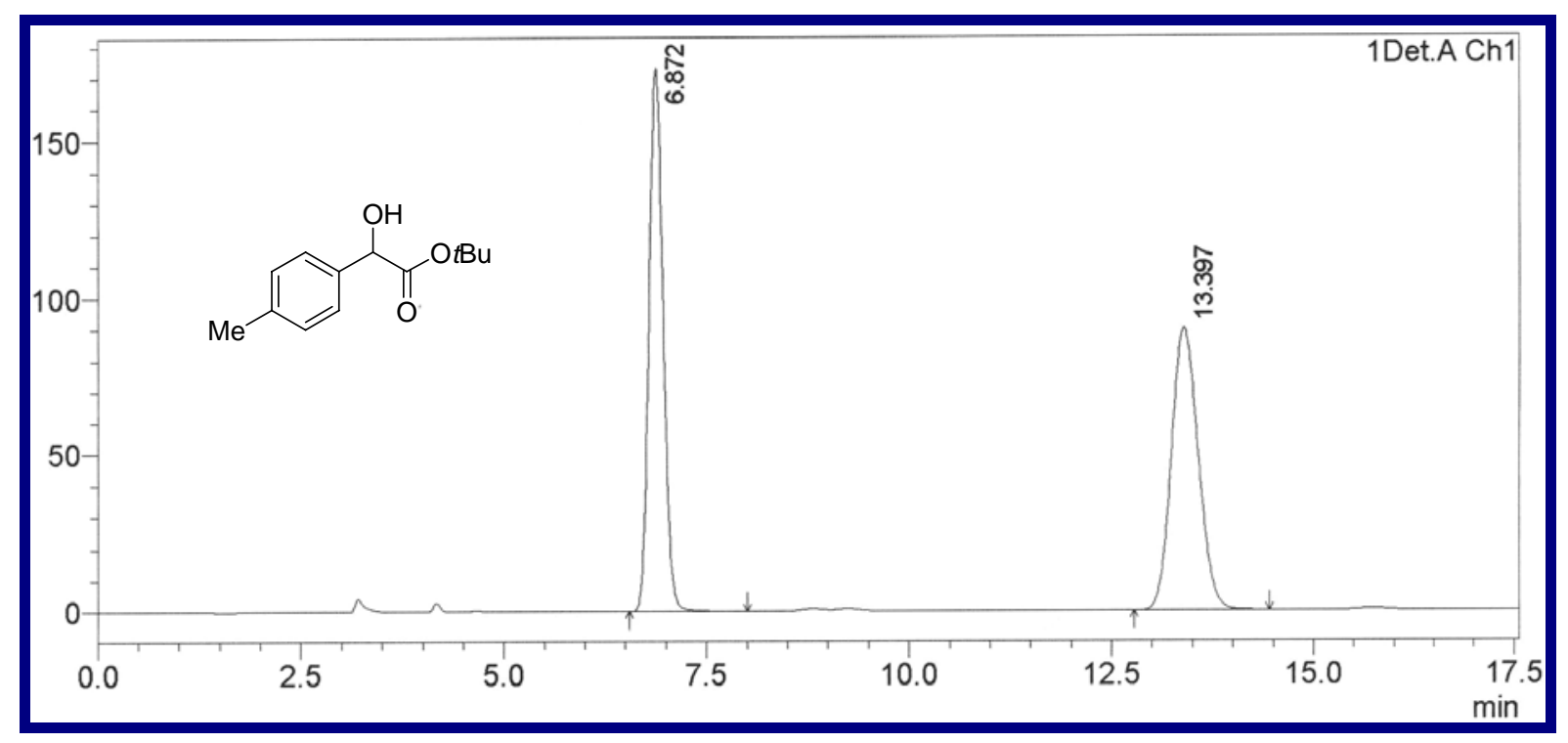

Detector A Ch1 220nm
\begin{tabular}{|l|r|r|}
\hline Peak\# & Ret. Time & Area $\%$ \\
\hline 1 & 6.872 & 50.221 \\
\hline 2 & 13.397 & 49.779 \\
\hline Total & & 100.000 \\
\hline
\end{tabular}

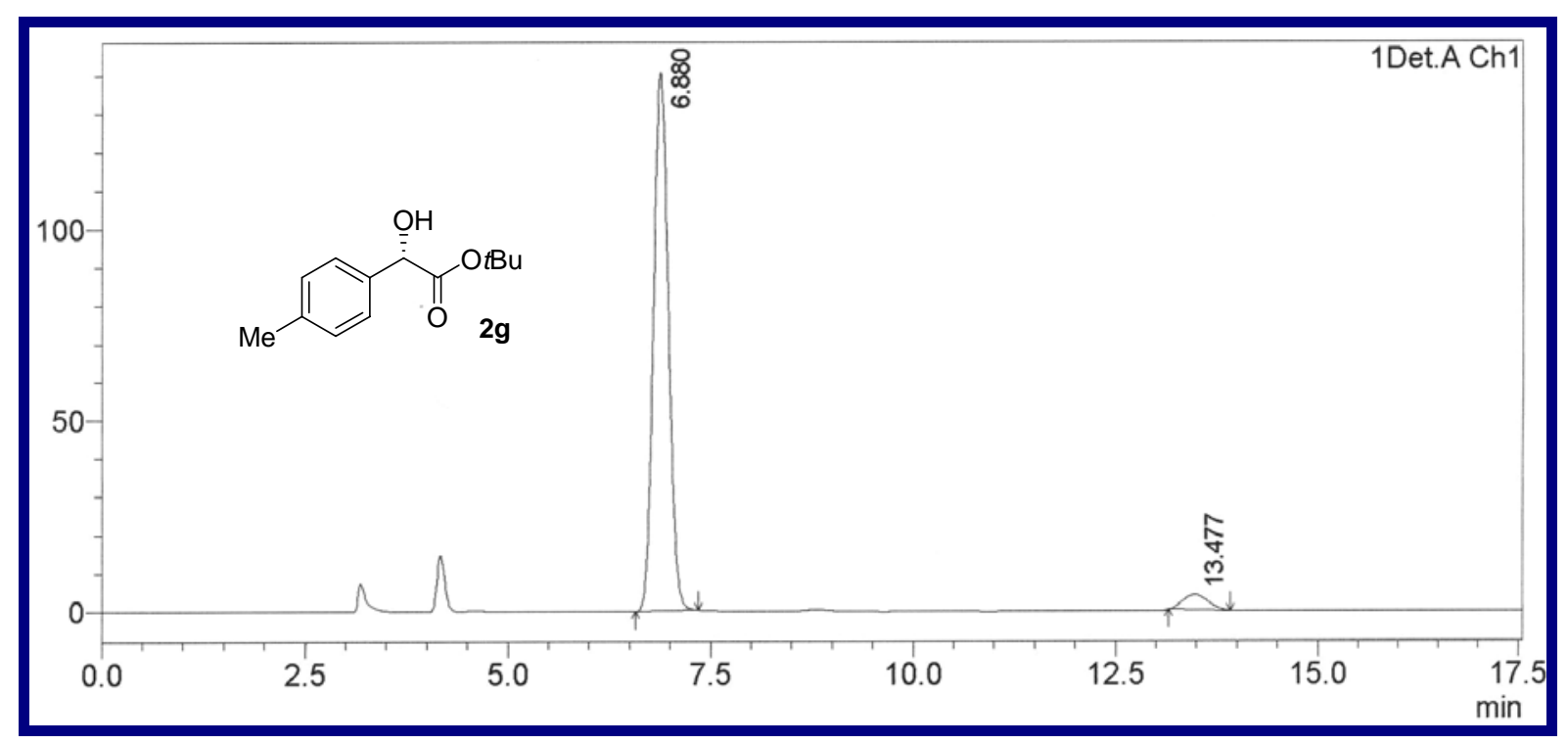

Detector A Ch1 220nm
\begin{tabular}{|l|r|r|}
\hline Peak \# & Ret. Time & \multicolumn{1}{|c|}{ Area $\%$} \\
\hline 1 & 6.880 & 95.538 \\
\hline 2 & 13.477 & 4.462 \\
\hline Total & & 100.000 \\
\hline
\end{tabular}




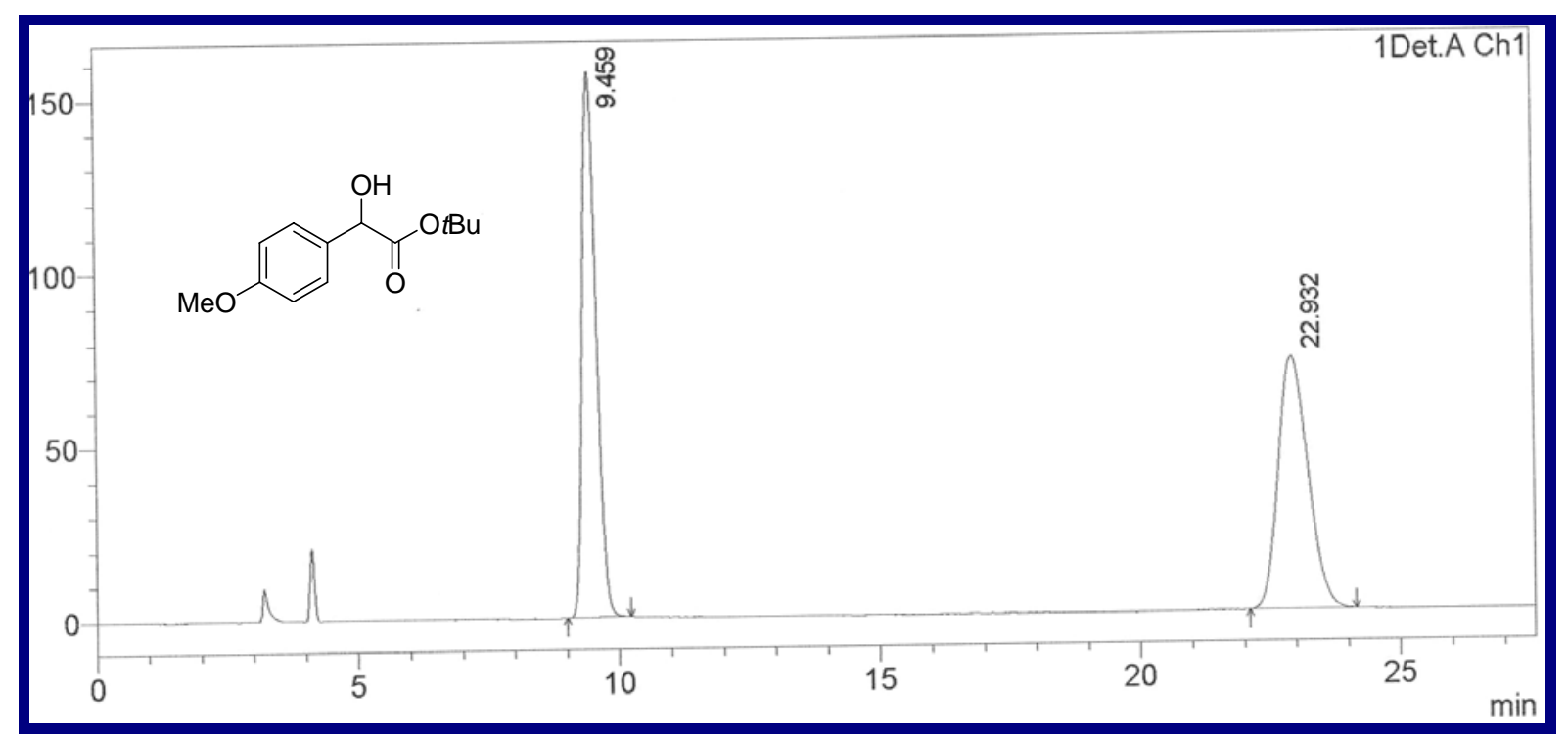

Detector A Ch1 220nm
\begin{tabular}{|l|r|r|}
\hline Peak \# & Ret. Time & Area $\%$ \\
\hline 1 & 9.459 & 50.050 \\
\hline 2 & 22.932 & 49.950 \\
\hline Total & & 100.000 \\
\hline
\end{tabular}

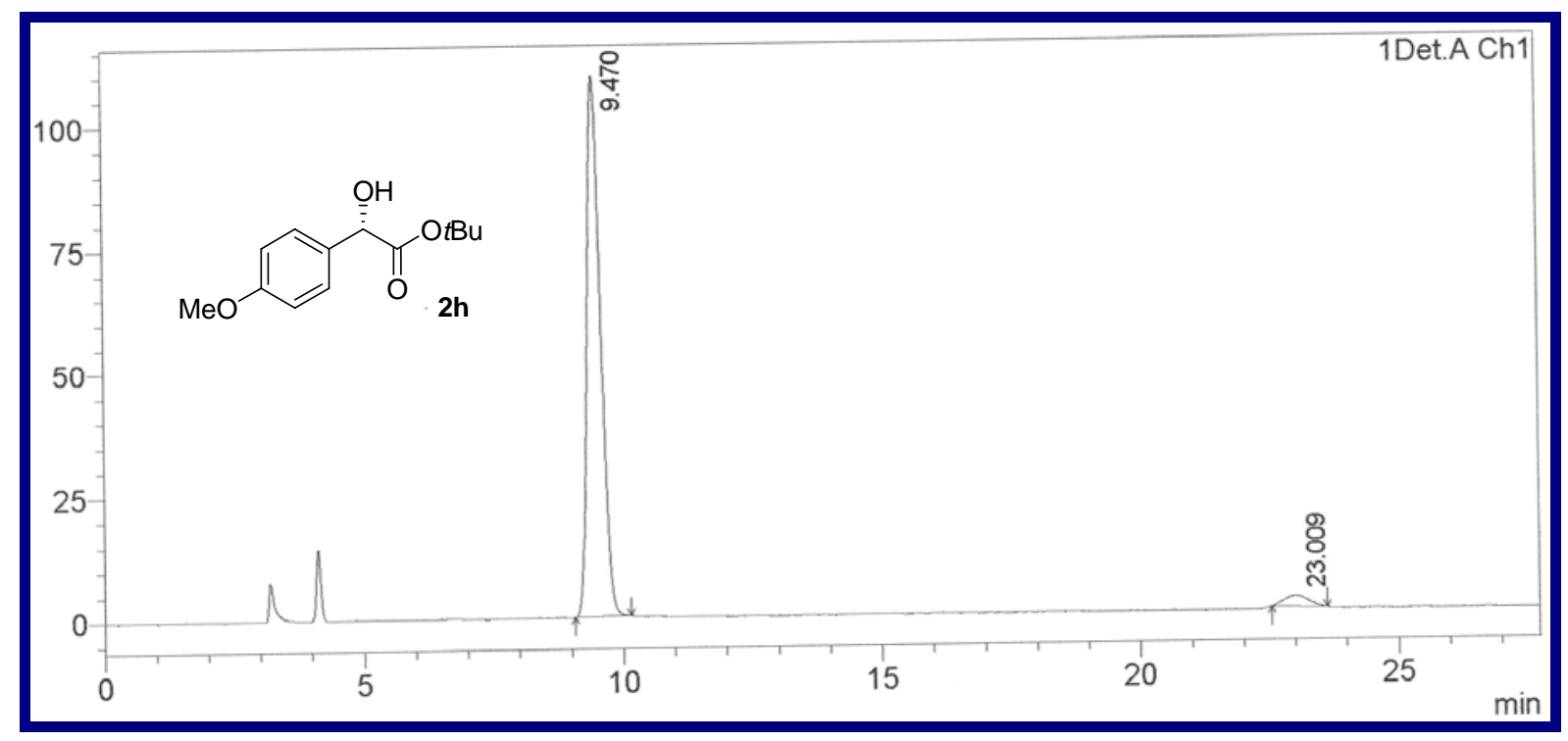

Detector A Ch1 220nm
\begin{tabular}{|l|r|r|}
\hline Peak \# & Ret. Time & Area $\%$ \\
\hline 1 & 9.470 & 96.350 \\
\hline 2 & 23.009 & 3.650 \\
\hline Total & & 100.000 \\
\hline
\end{tabular}




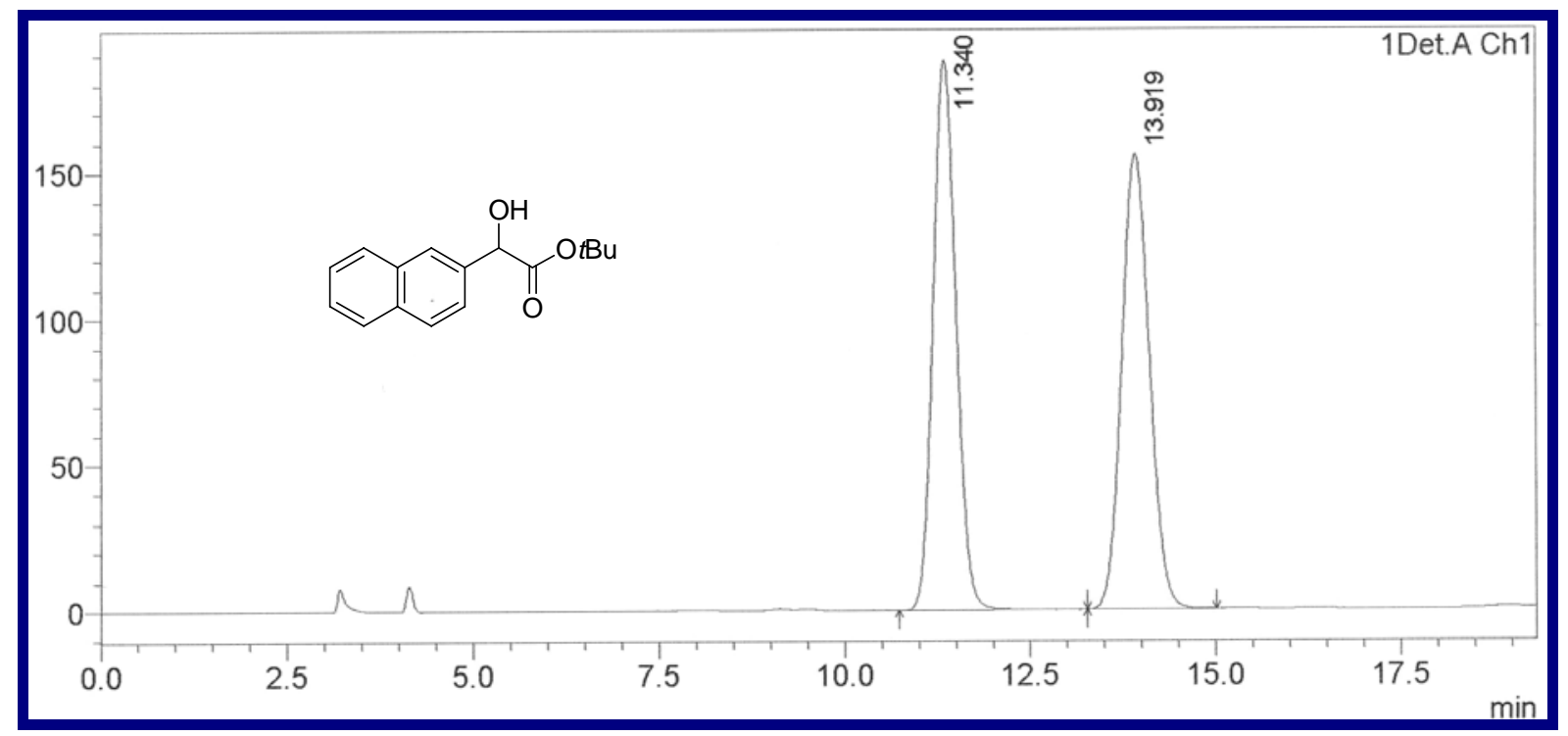

Detector A Ch1 220nm
\begin{tabular}{|l|r|r|}
\hline Peak \# & Ret. Time & Area $\%$ \\
\hline 1 & 11.340 & 50.021 \\
\hline 2 & 13.919 & 49.979 \\
\hline Total & & 100.000 \\
\hline
\end{tabular}

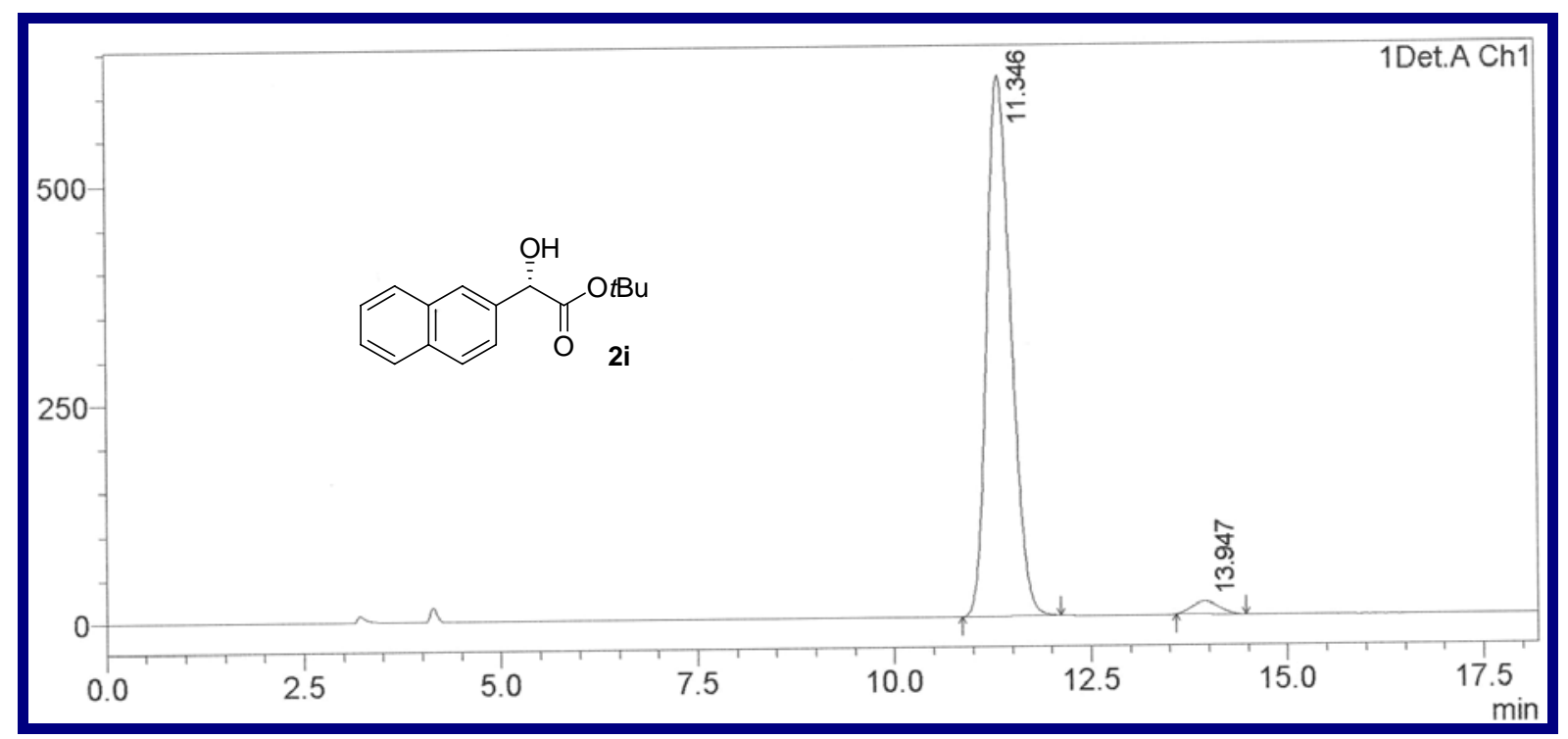

Detector A Ch1 220nm
\begin{tabular}{|l|r|r|}
\hline Peak \# & Ret. Time & \multicolumn{1}{|c|}{ Area $\%$} \\
\hline 1 & 11.346 & 97.432 \\
\hline 2 & 13.947 & 2.568 \\
\hline Total & & 100.000 \\
\hline
\end{tabular}




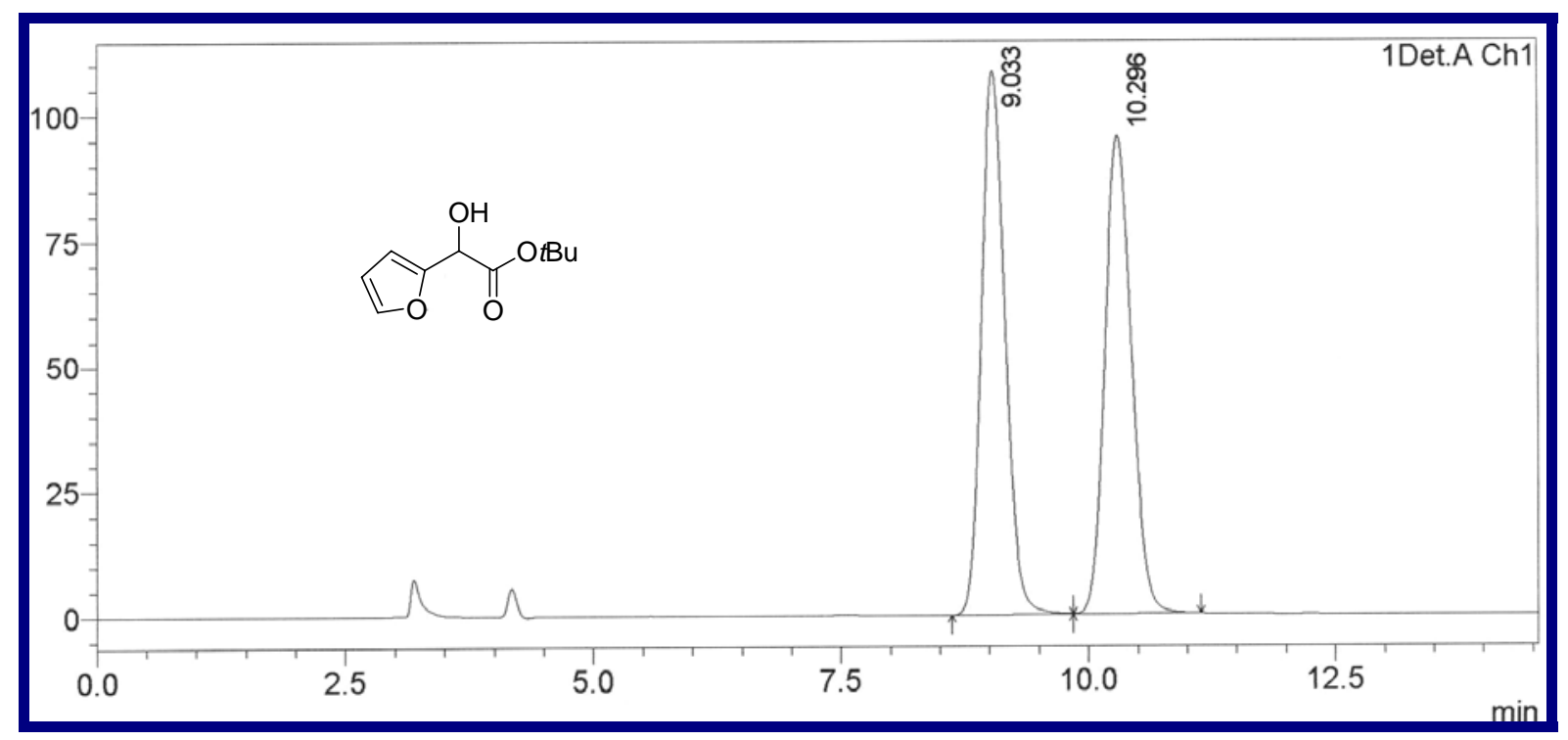

Detector A Ch1 220nm
\begin{tabular}{|l|r|r|}
\hline Peak \# & Ret. Time & \multicolumn{1}{|c|}{ Area $\%$} \\
\hline 1 & 9.033 & 50.276 \\
\hline 2 & 10.296 & 49.724 \\
\hline Total & & 100.000 \\
\hline
\end{tabular}

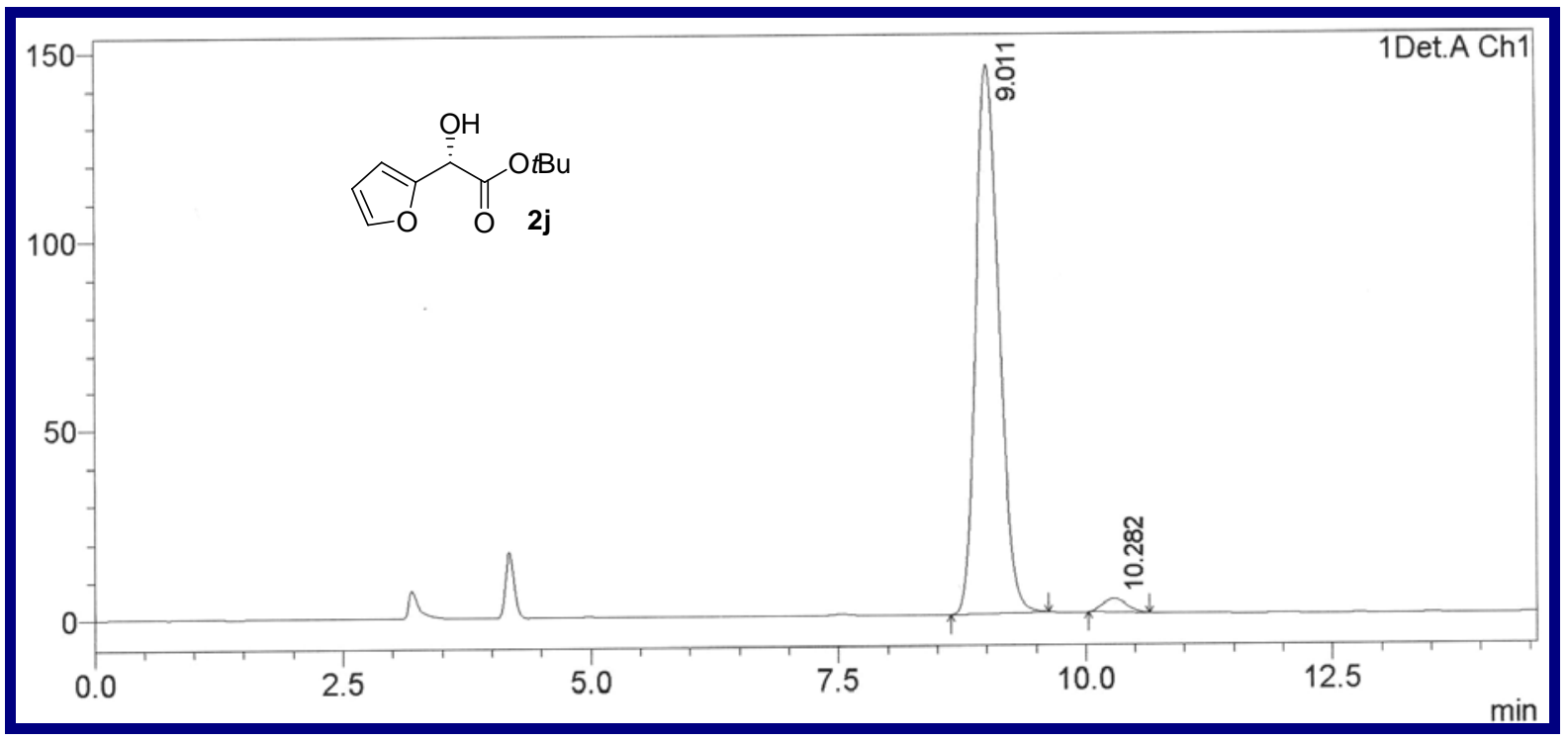

Detector A Ch1 220nm
\begin{tabular}{|l|r|r|}
\hline Peak \# & Ret. Time & \multicolumn{1}{|c|}{ Area $\%$} \\
\hline 1 & 9.011 & 97.463 \\
\hline 2 & 10.282 & 2.537 \\
\hline Total & & 100.000 \\
\hline
\end{tabular}




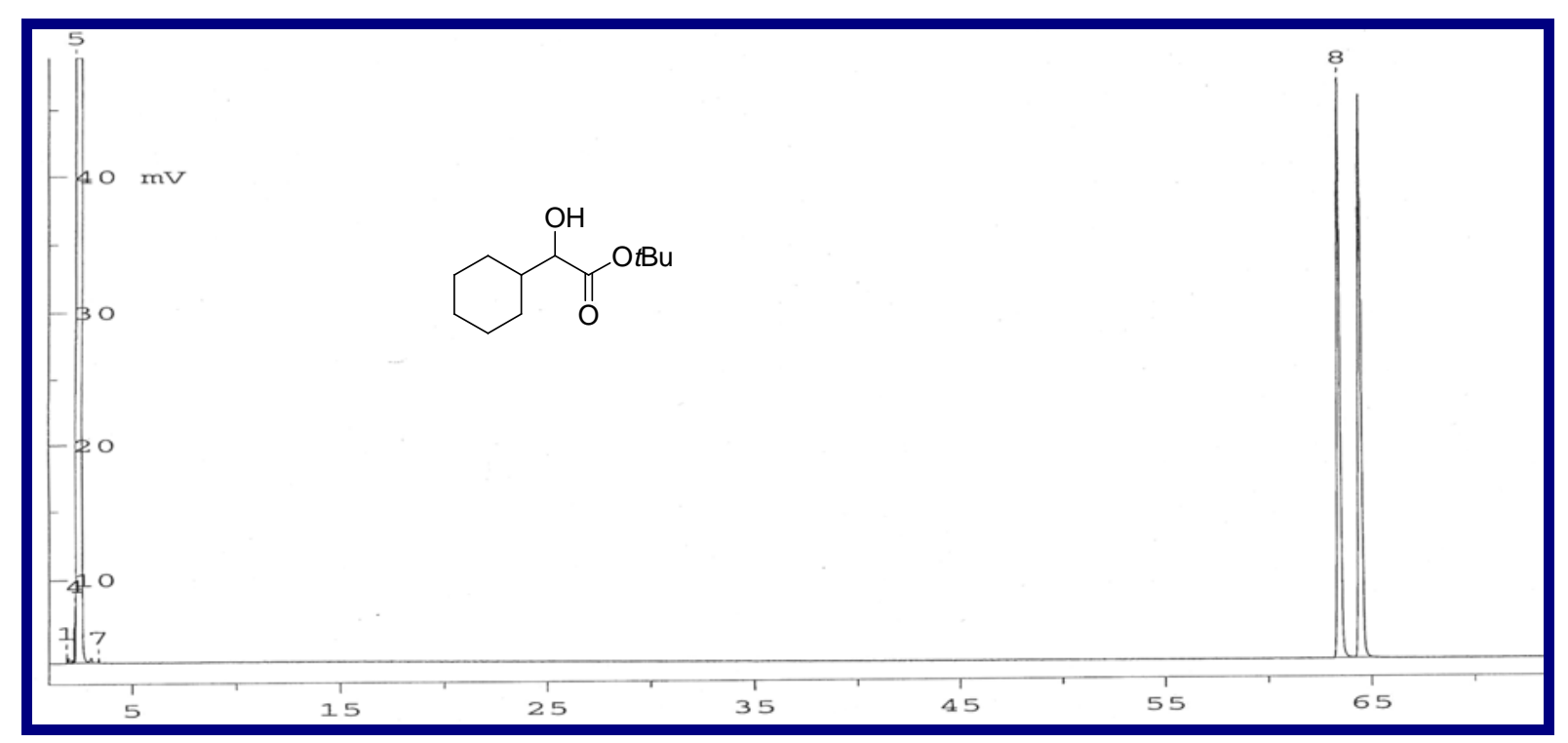

\begin{tabular}{|lll|} 
No. & $\min$. & area-\% \\
8 & 63.43 & 50.0 \\
9 & 64.45 & 50.0 \\
\hline
\end{tabular}

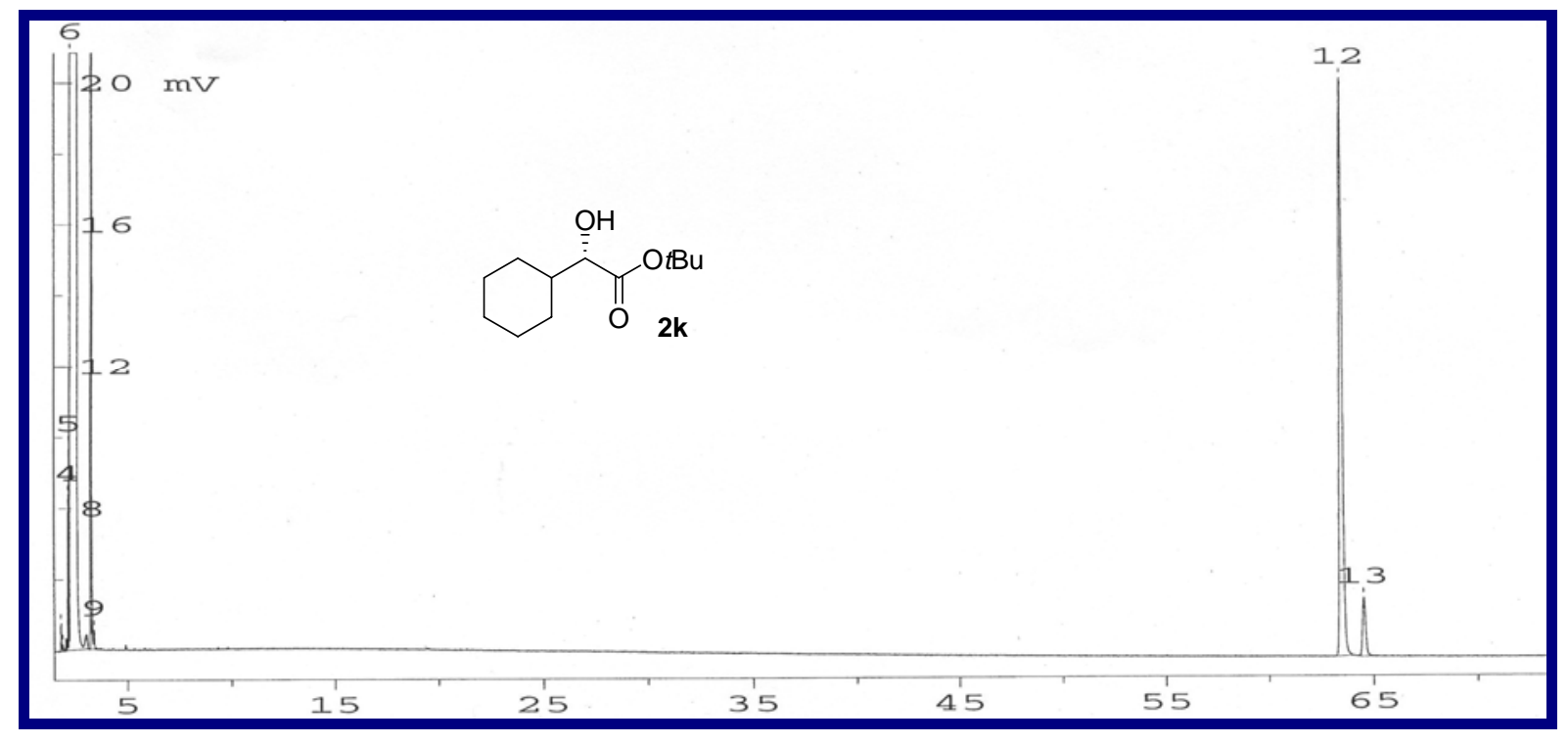

\begin{tabular}{ccc} 
No. & min. & area-\% \\
12 & 63.47 & 90.6 \\
13 & 64.53 & 9.43 \\
\hline
\end{tabular}




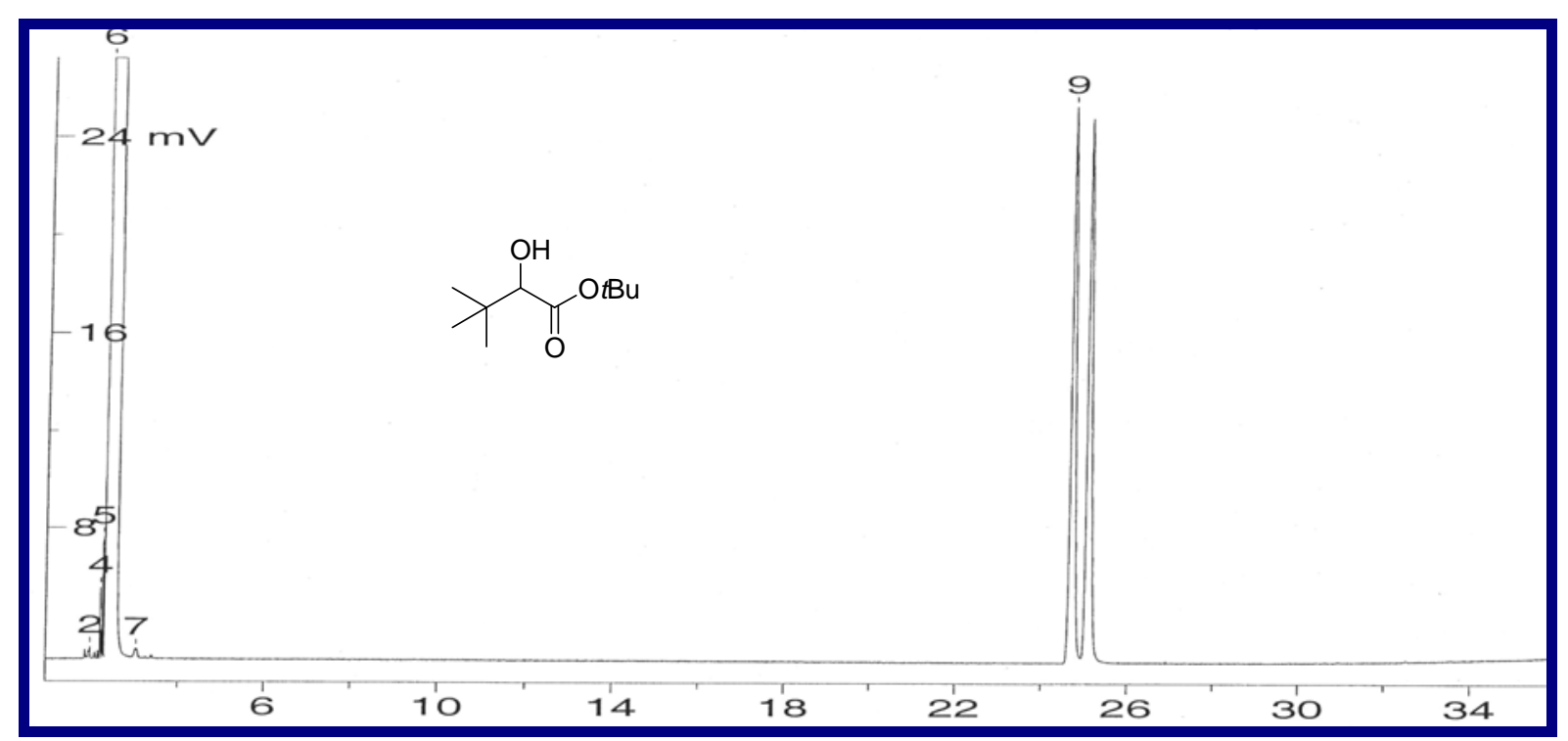

\begin{tabular}{|rcl|}
\hline No. & min. & area-\% \\
9 & 24.70 & 49.7 \\
10 & 25.09 & 50.3 \\
\hline
\end{tabular}

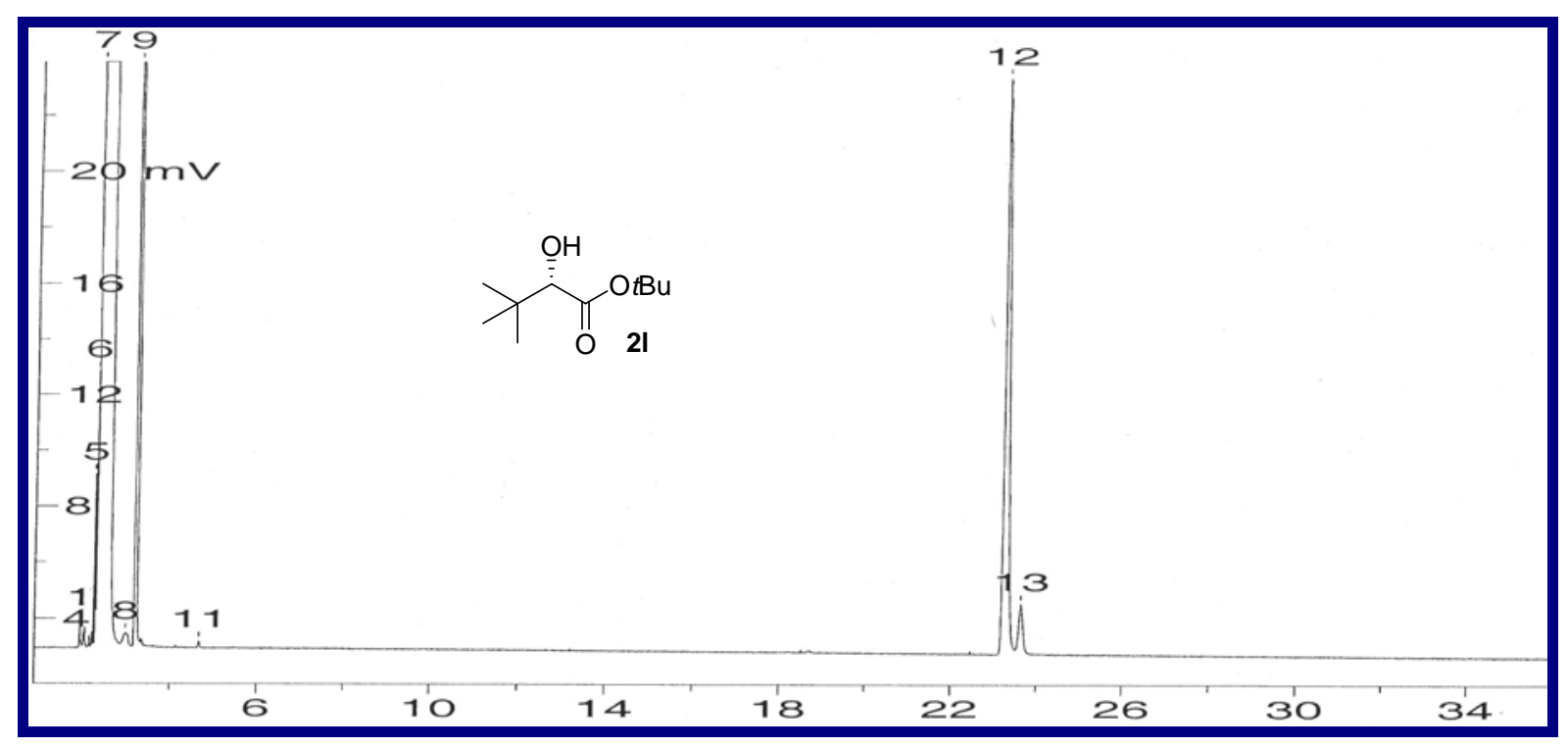

\begin{tabular}{ccc}
\hline No. & min. & area-9 \\
& & \\
12 & 23.27 & 92.0 \\
13 & 23.64 & 8.04 \\
\hline
\end{tabular}


- 19 -
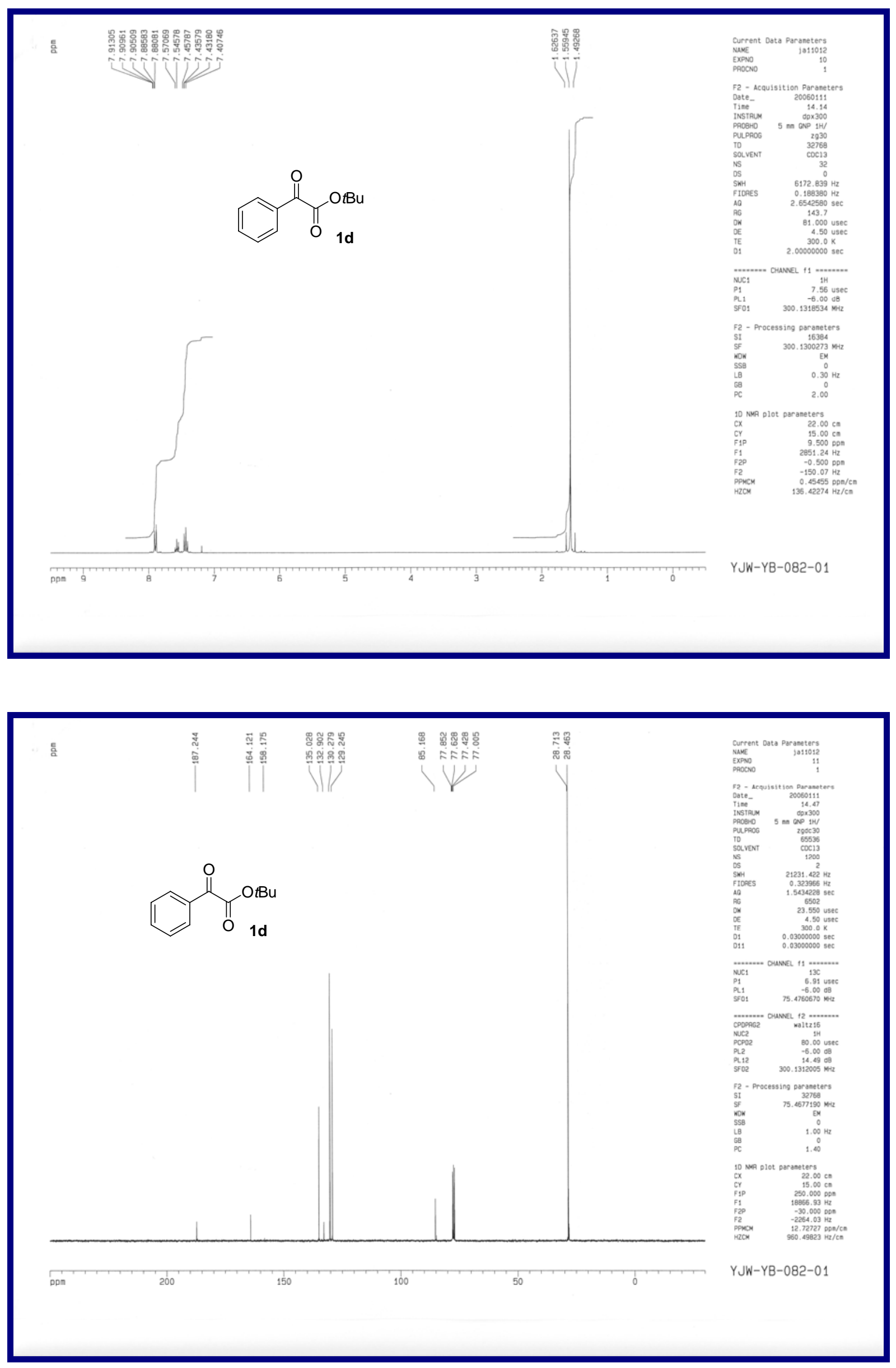

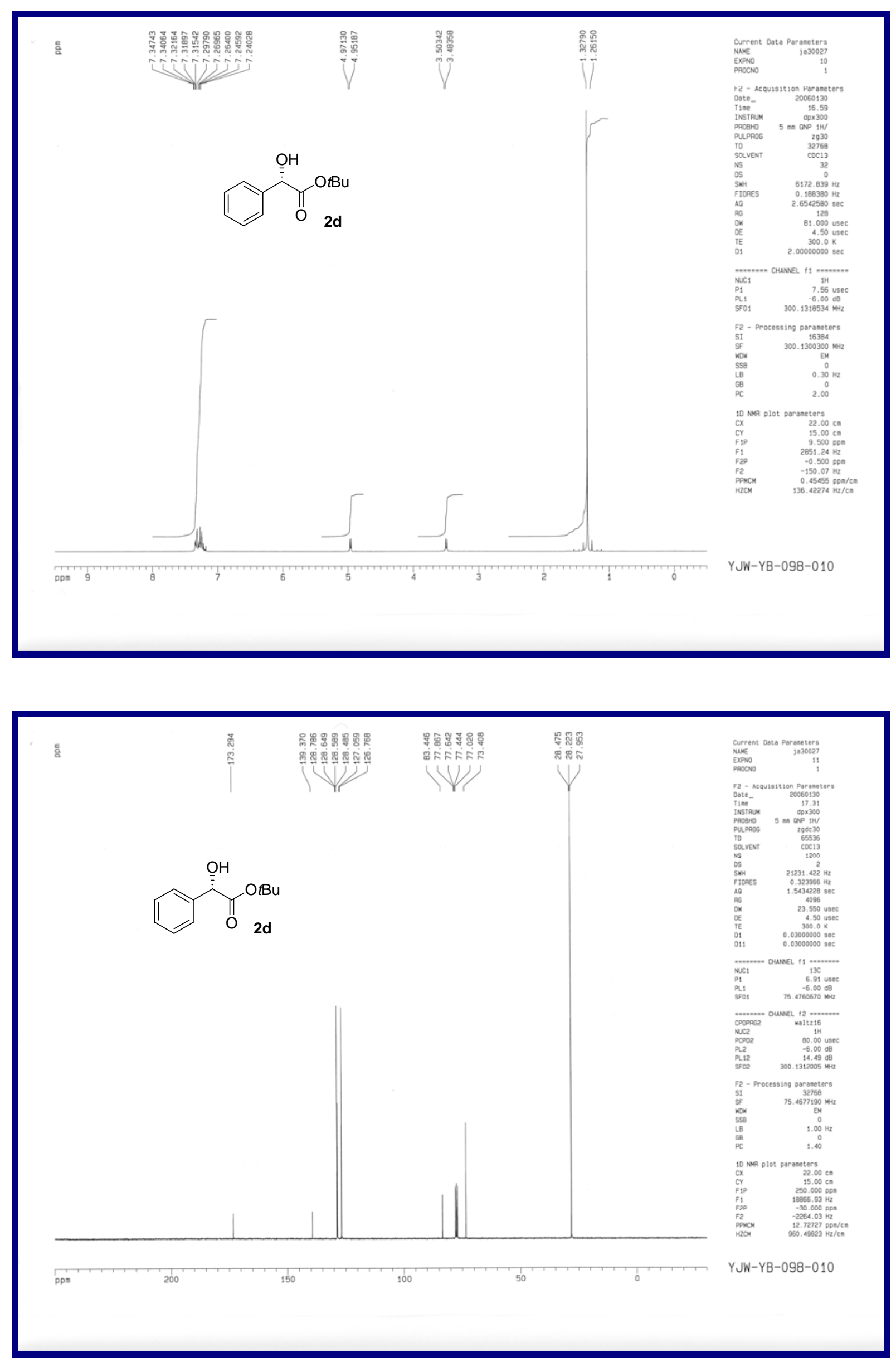

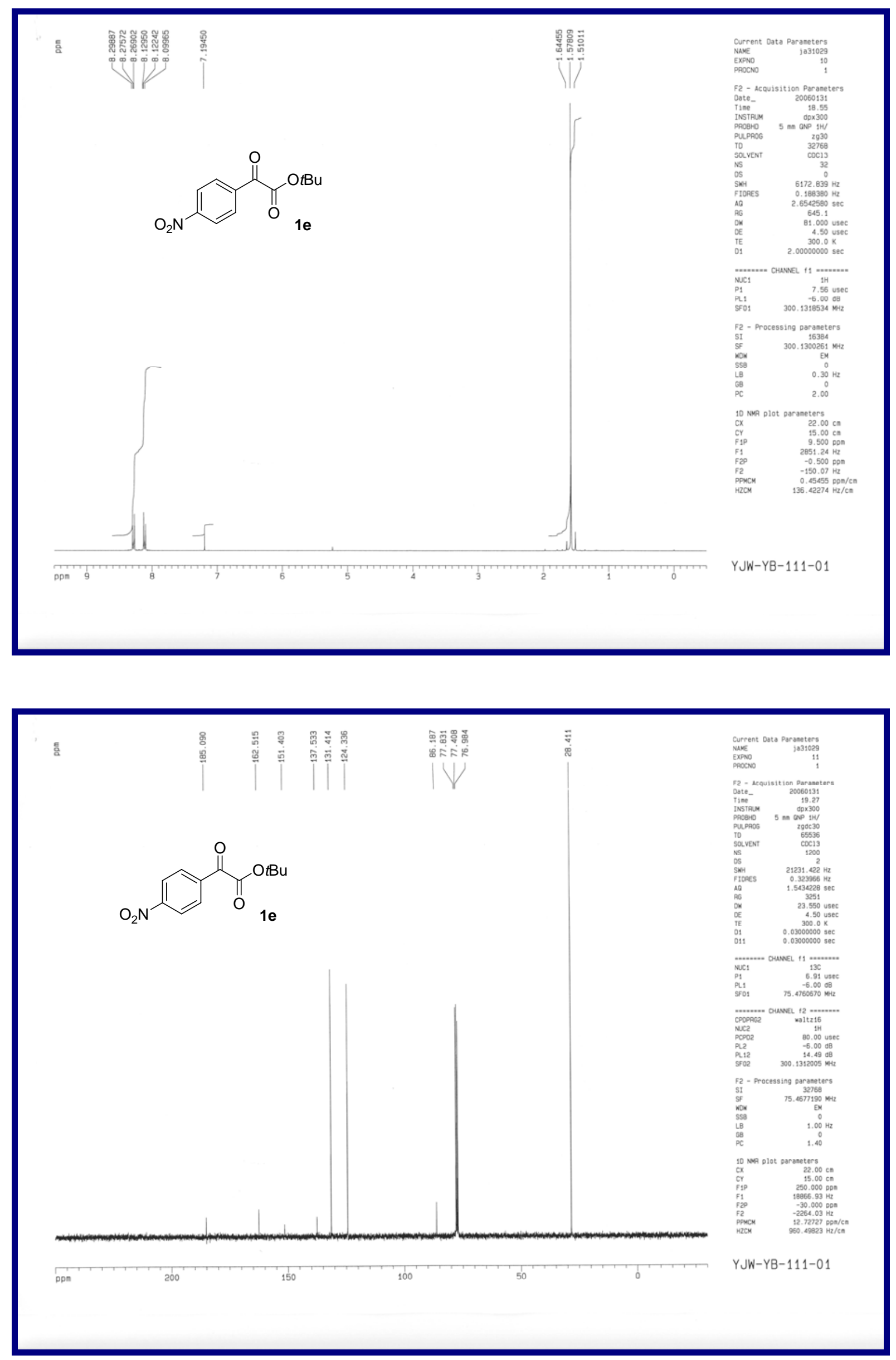

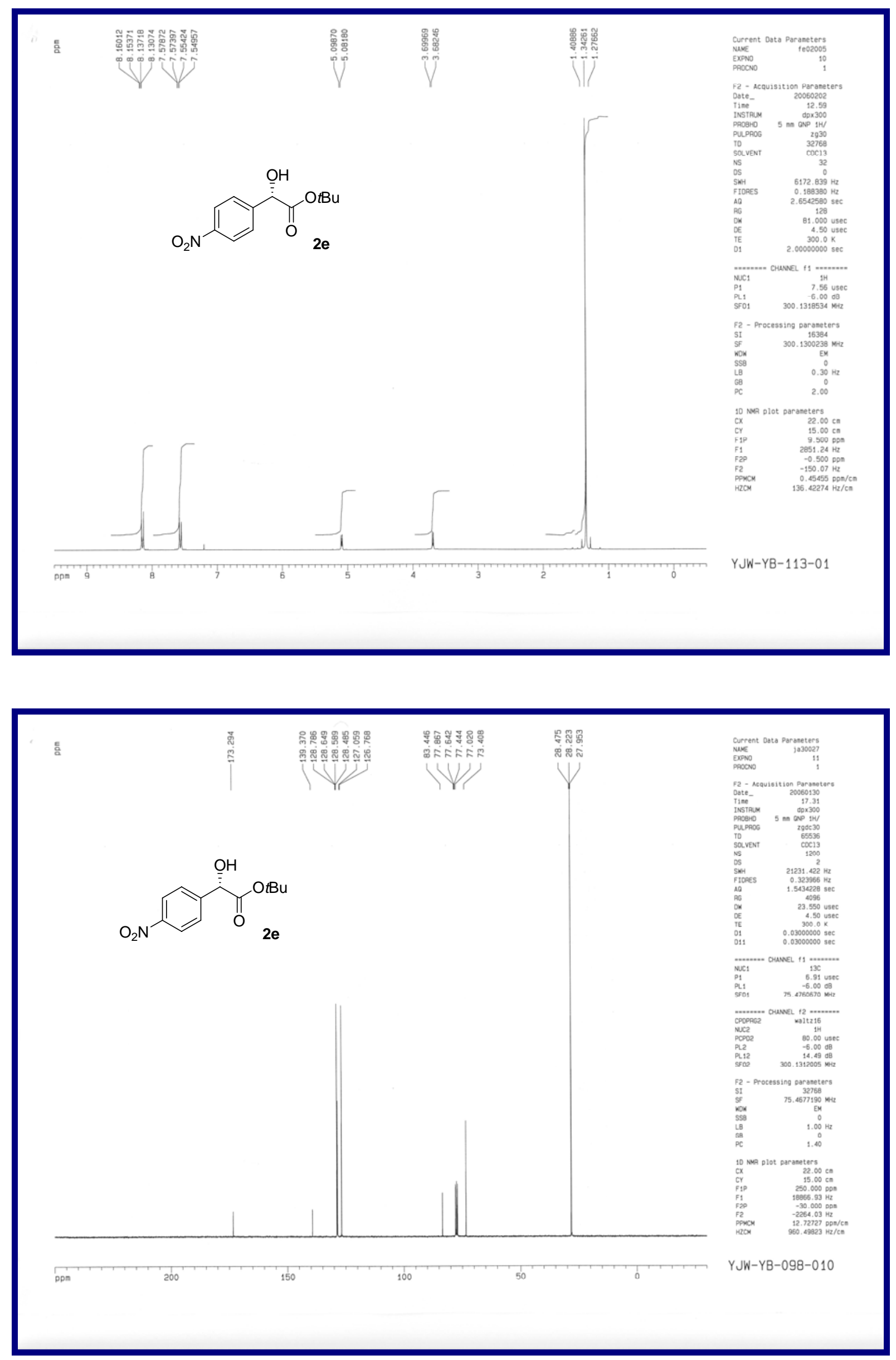

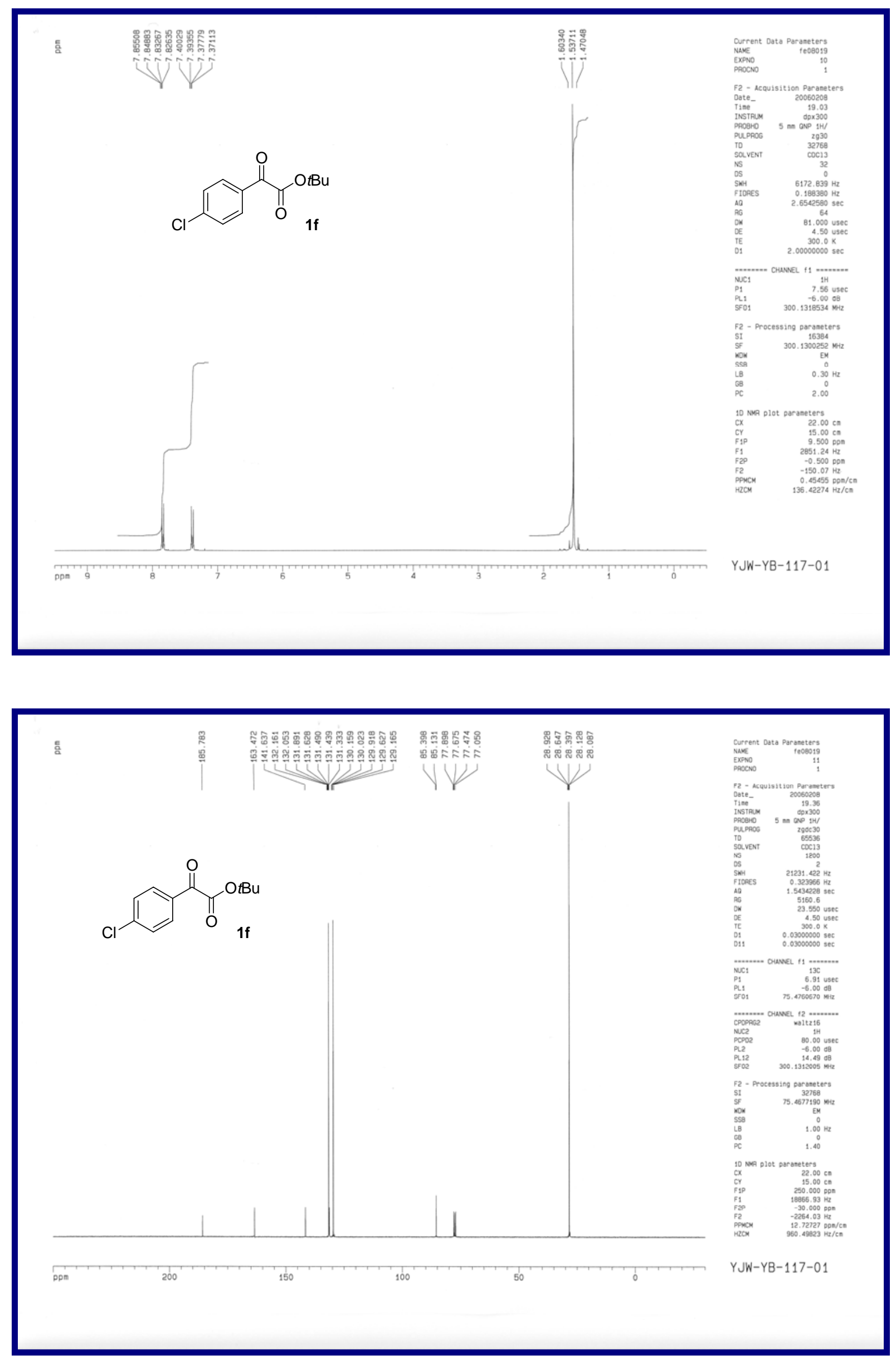

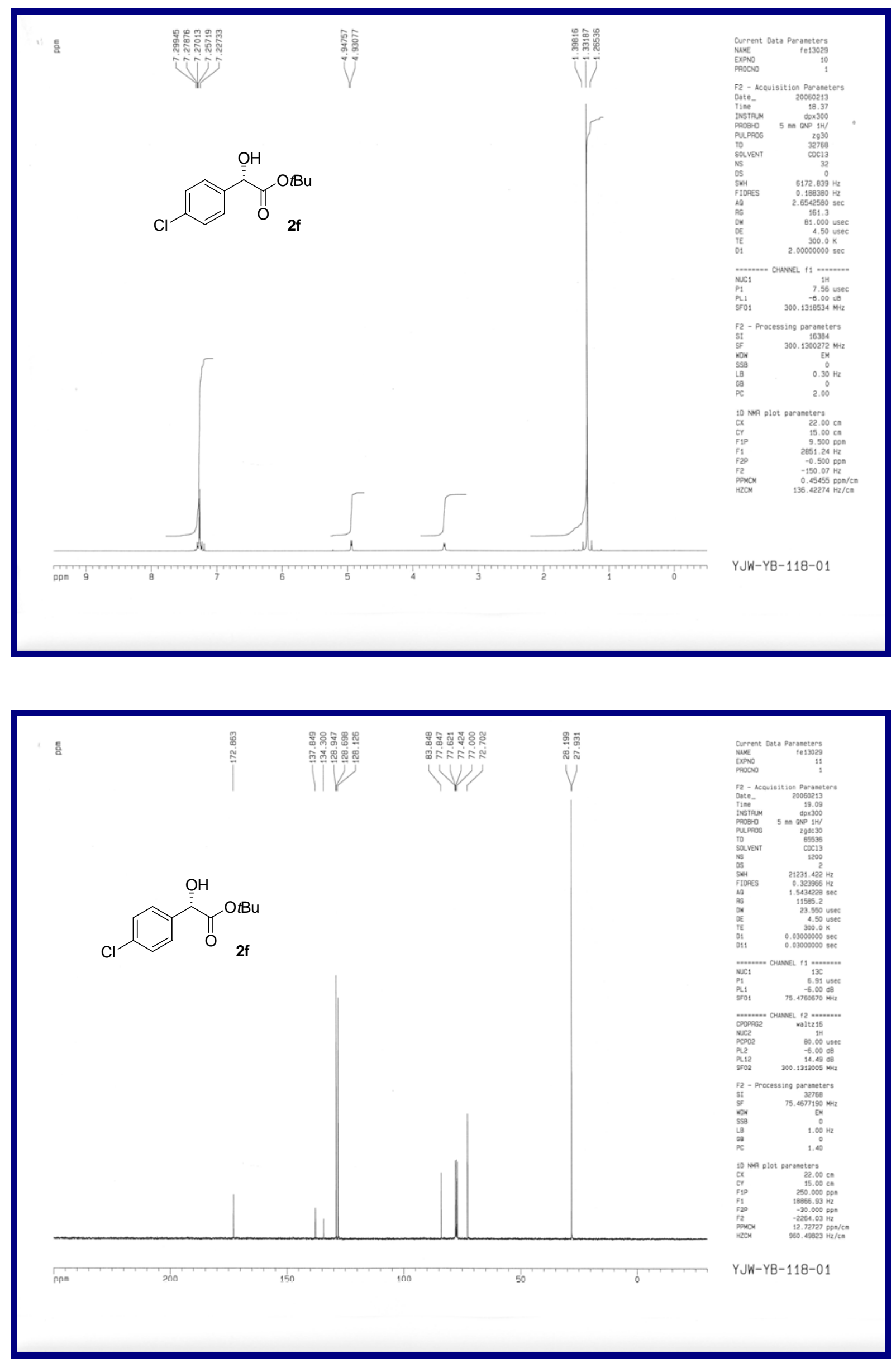

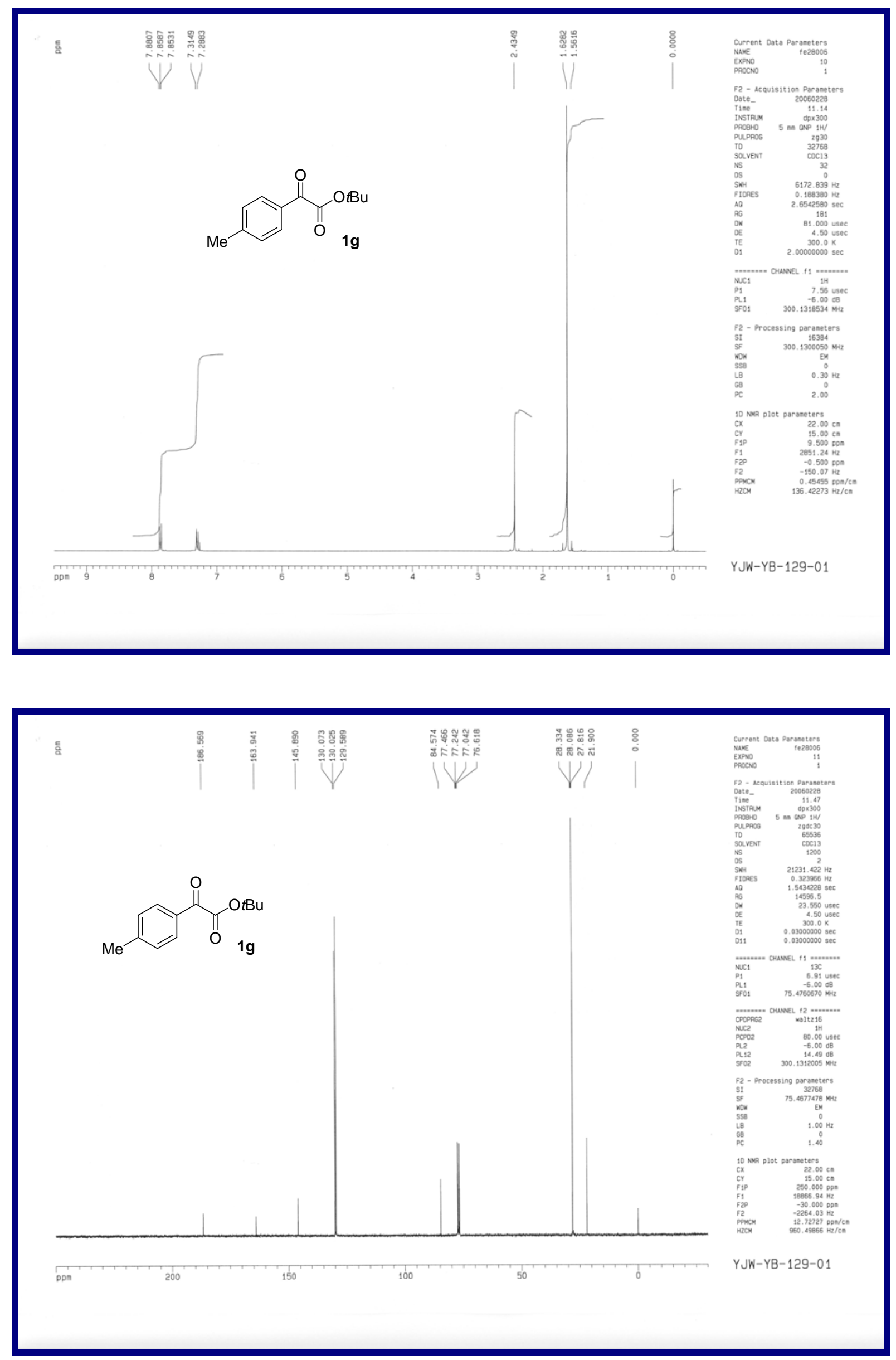

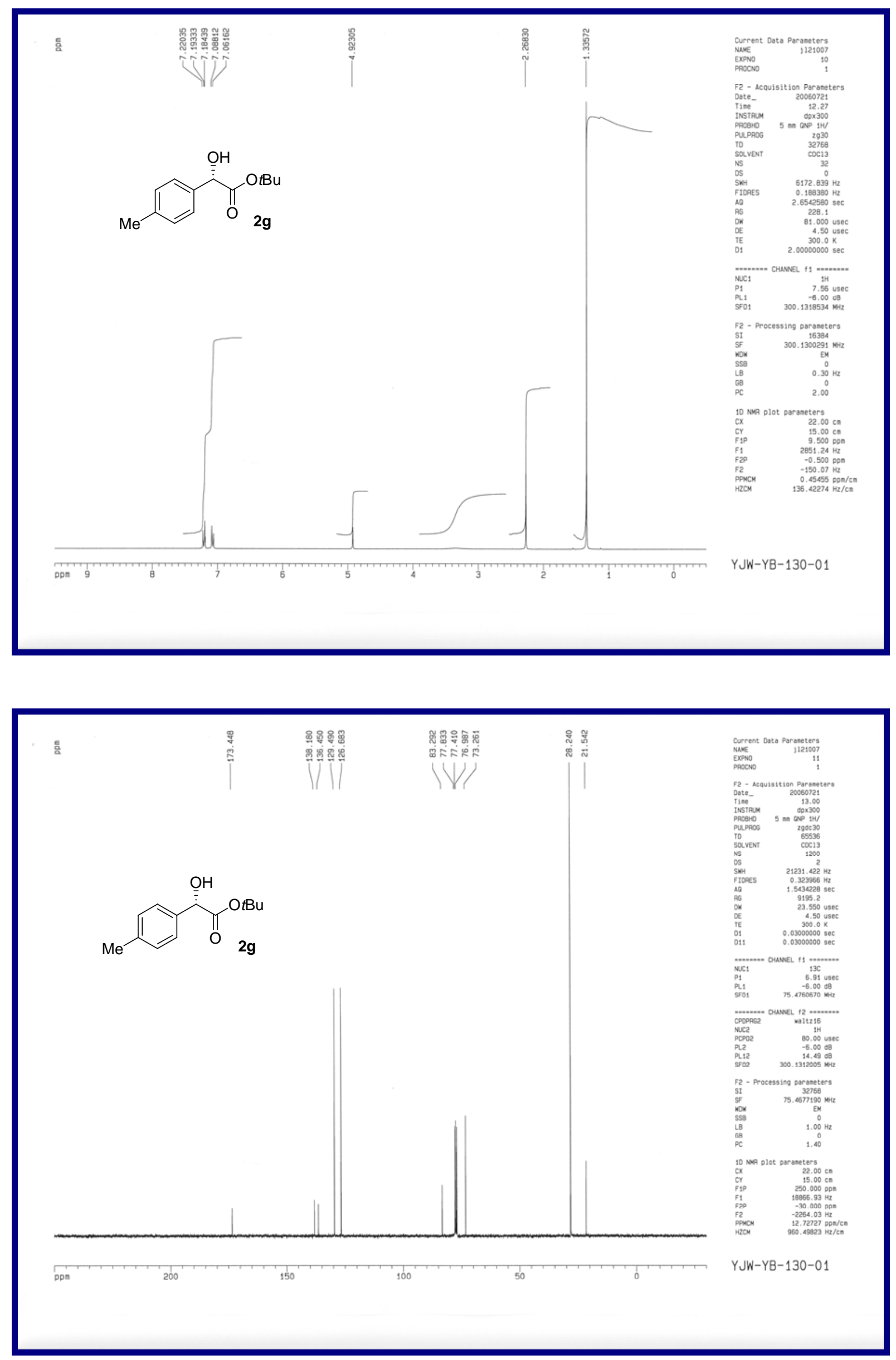

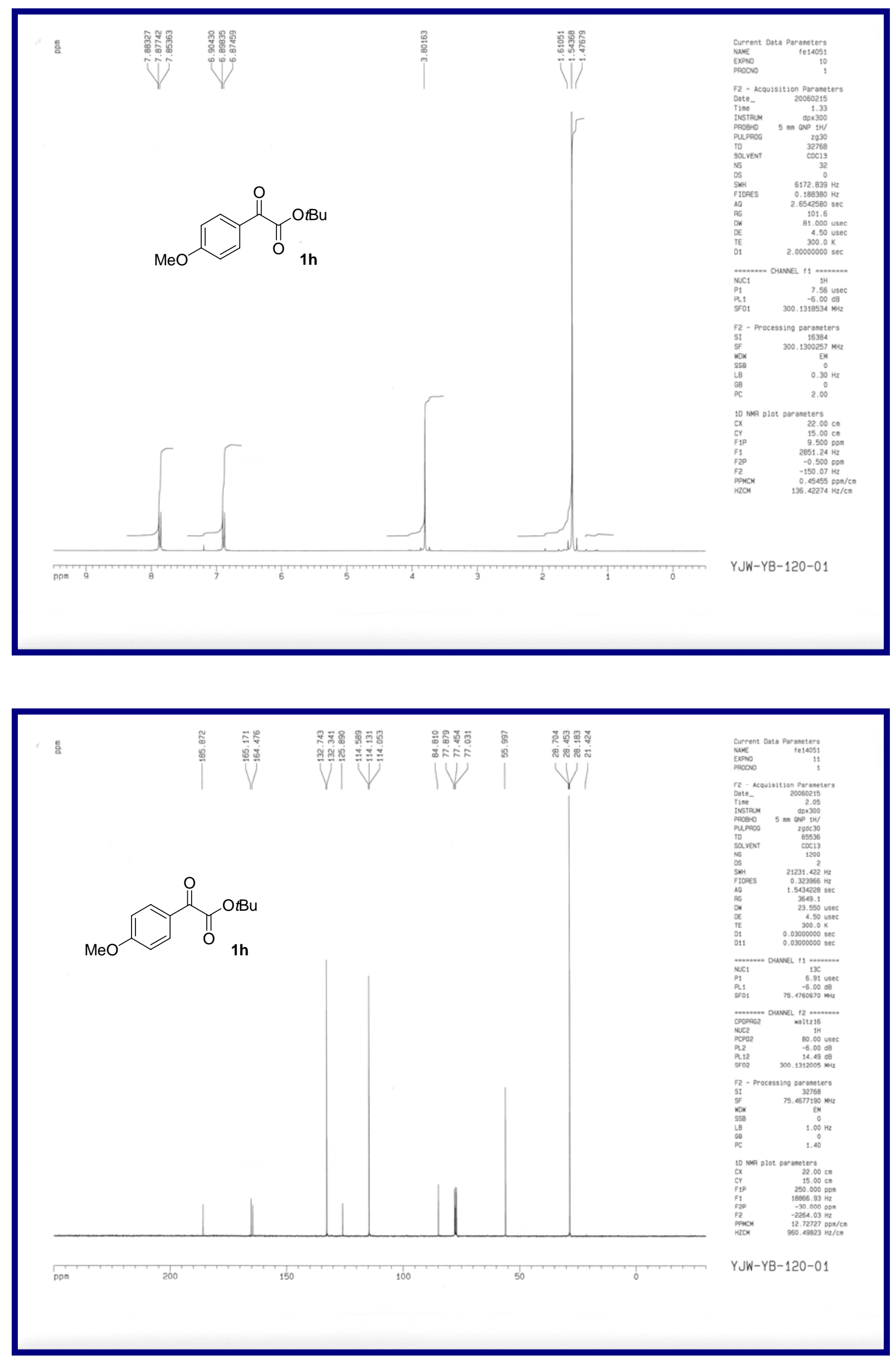

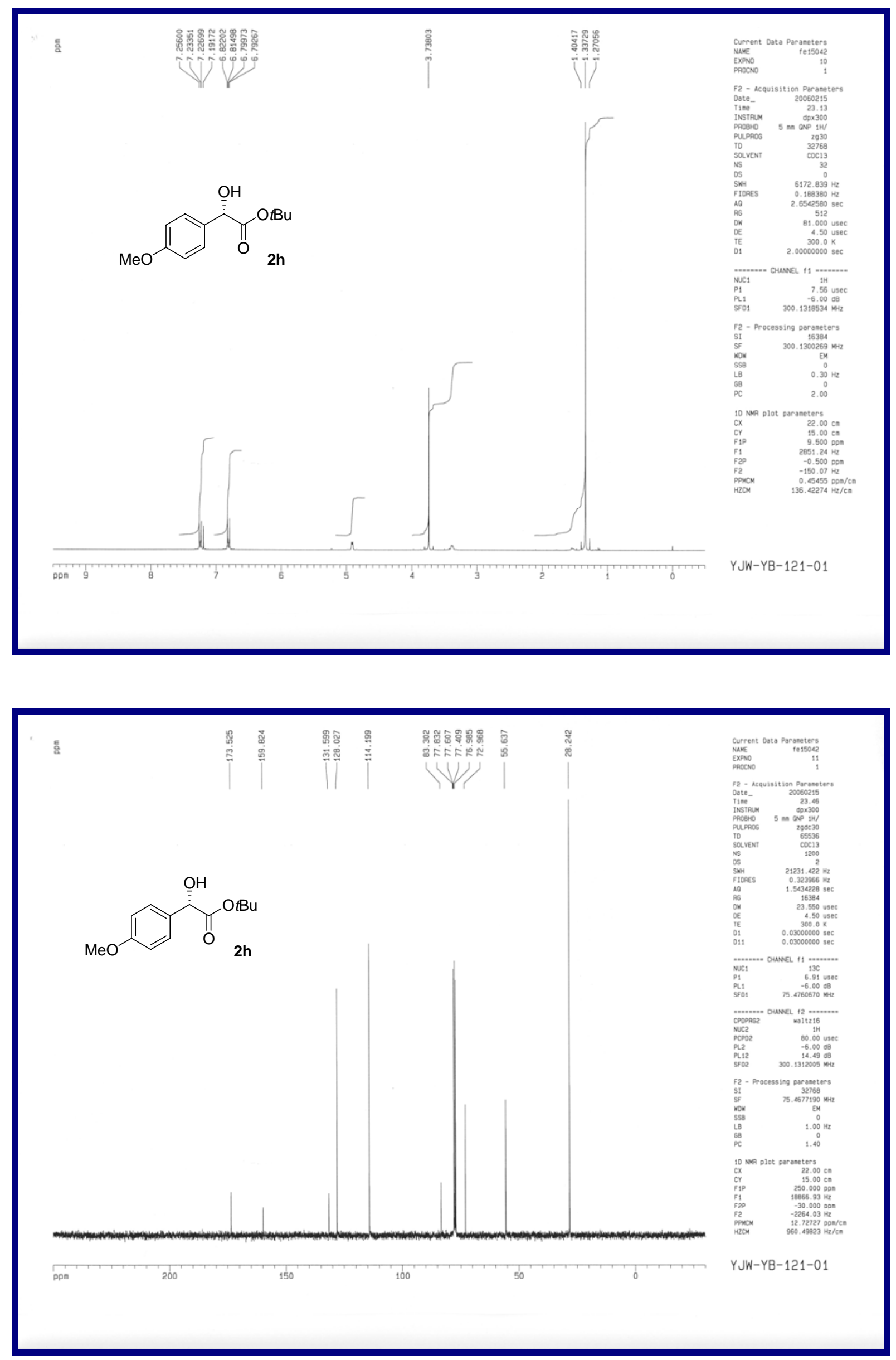

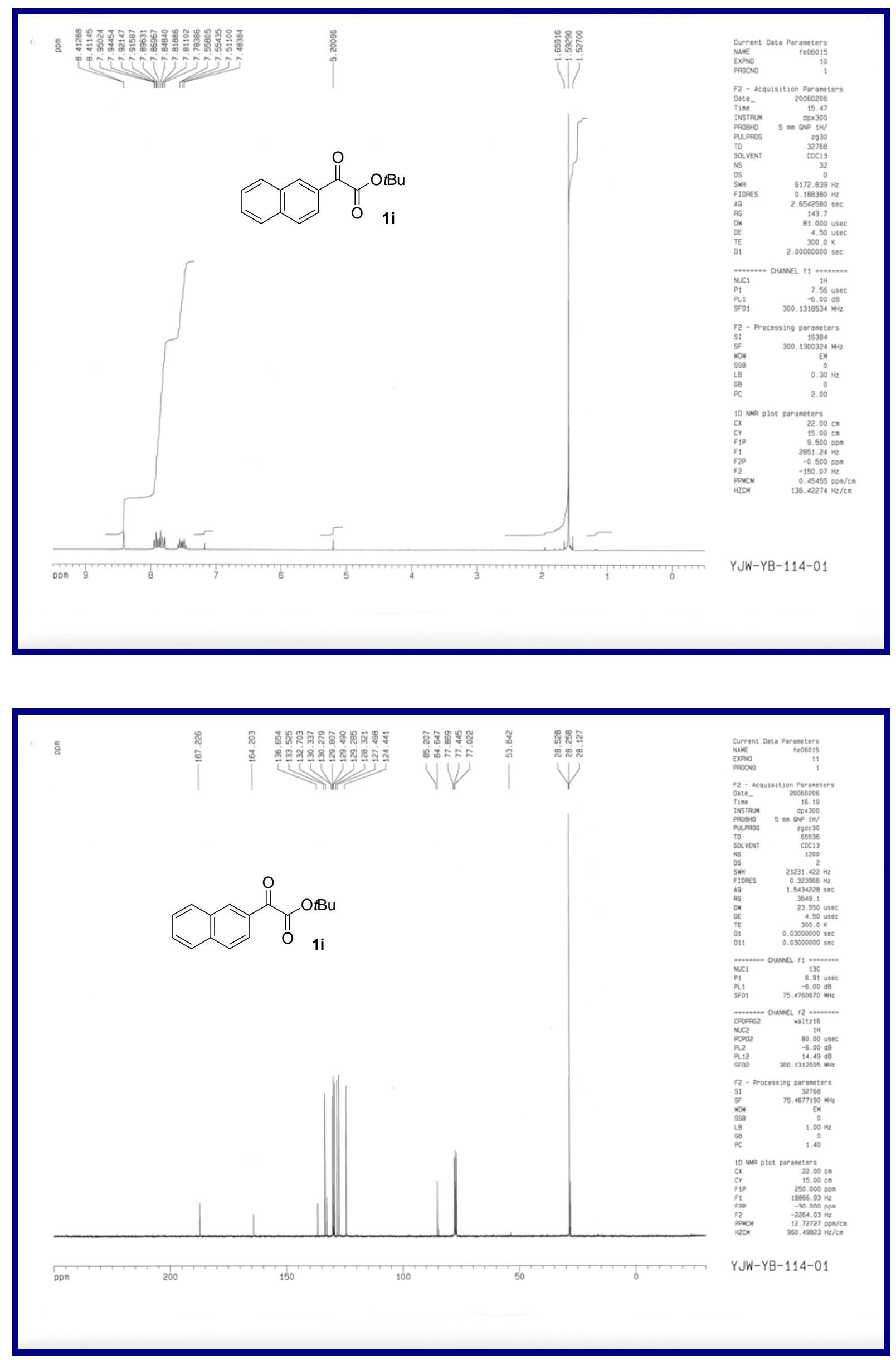

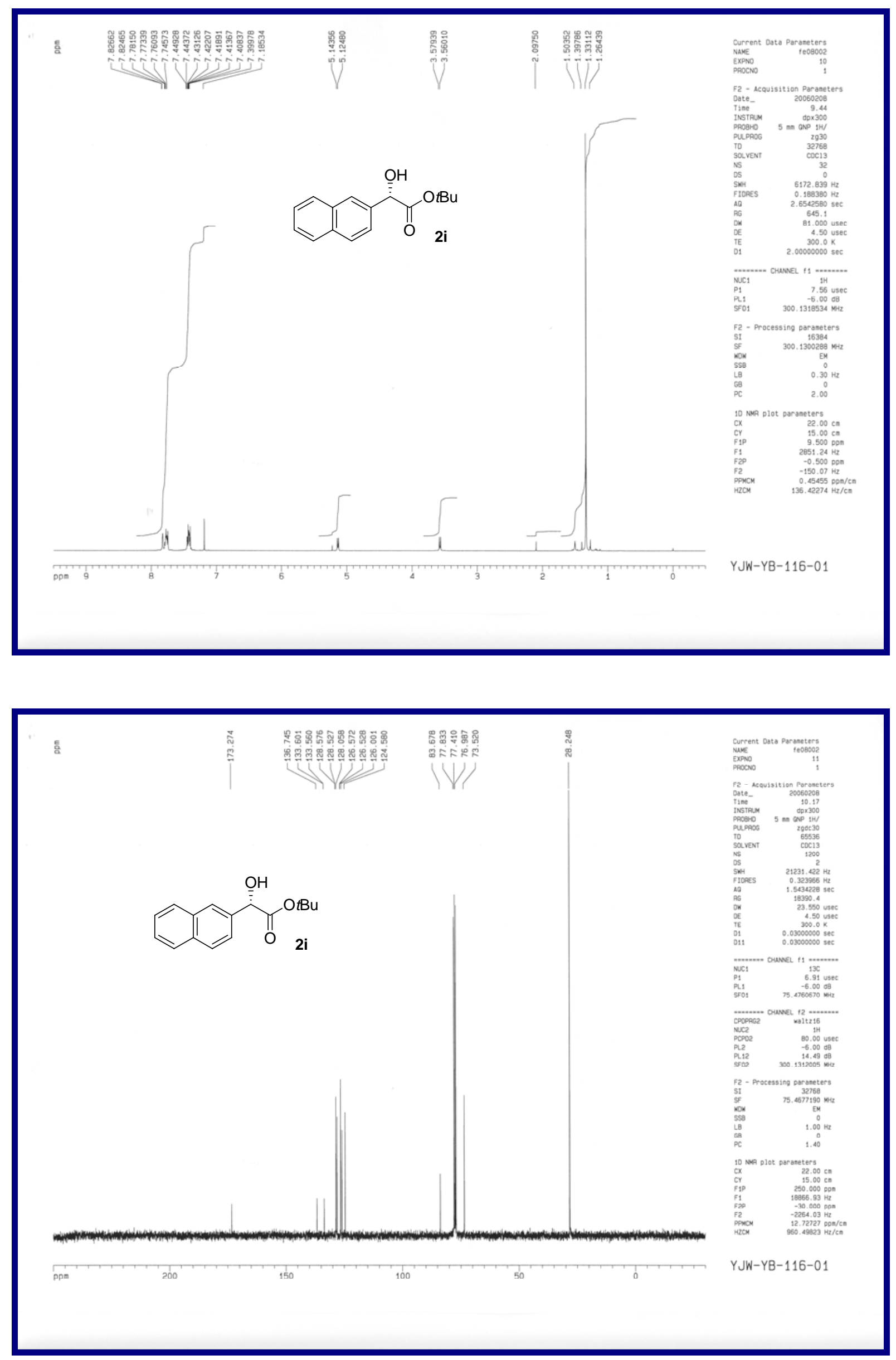

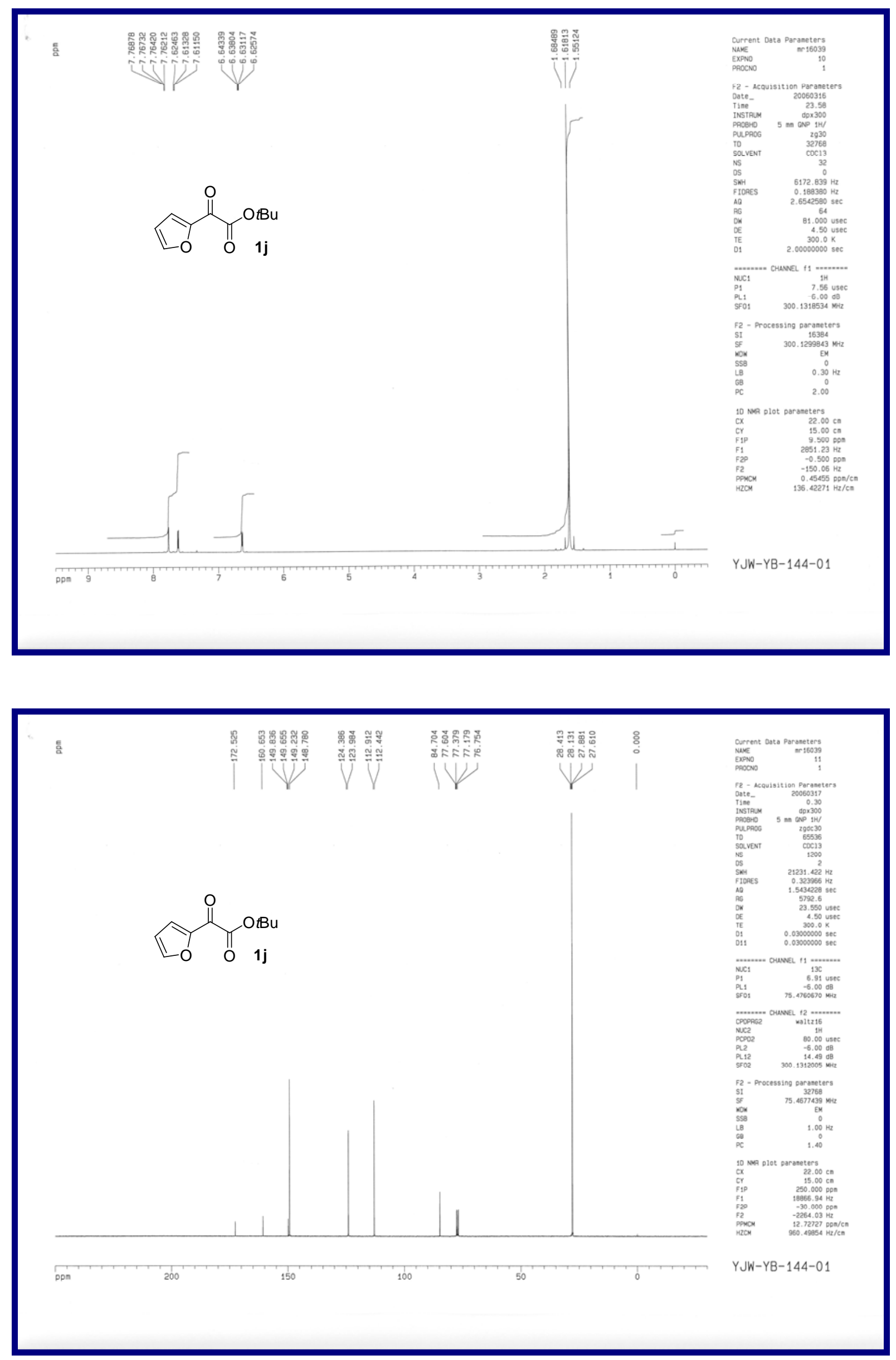

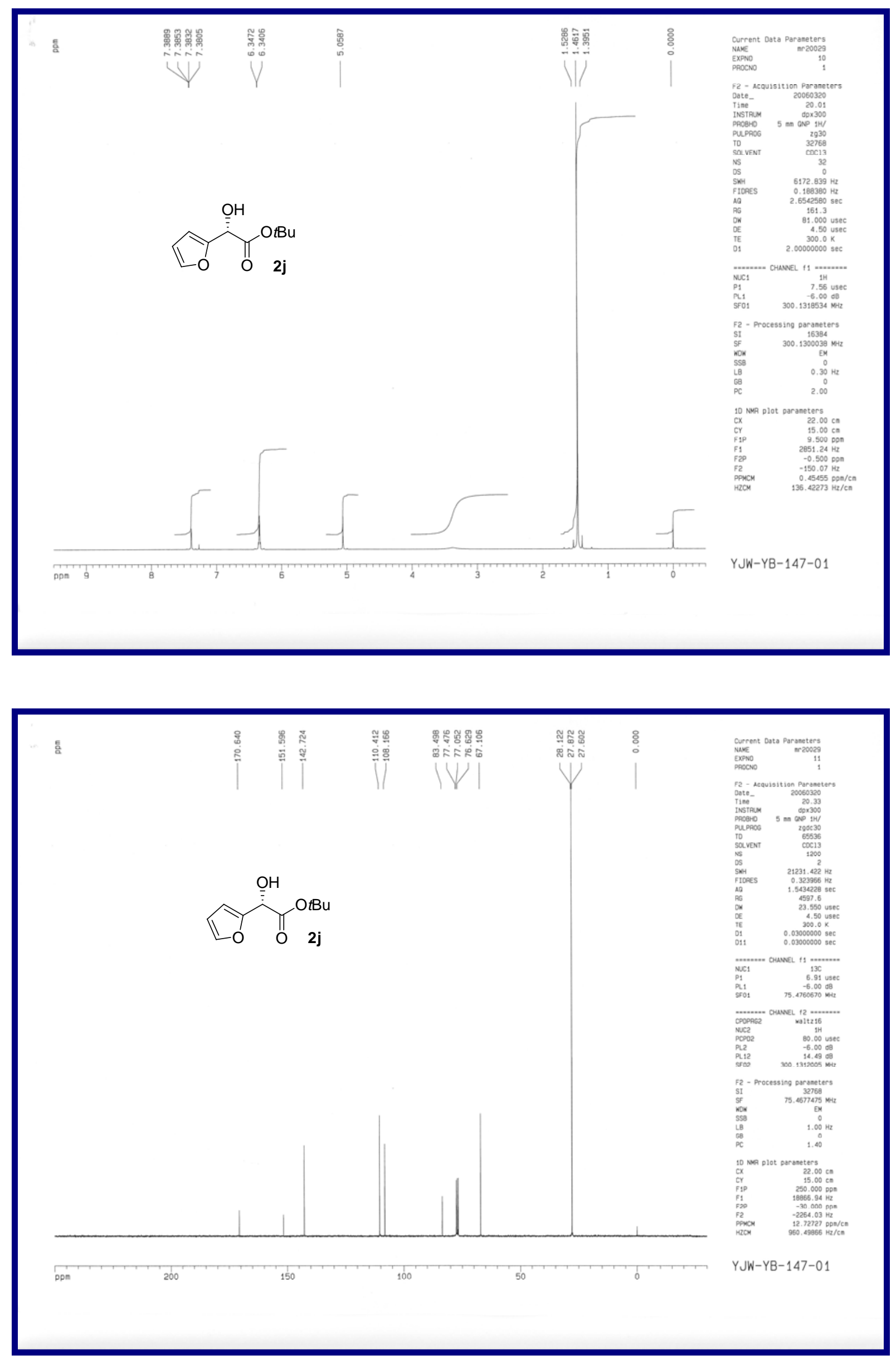

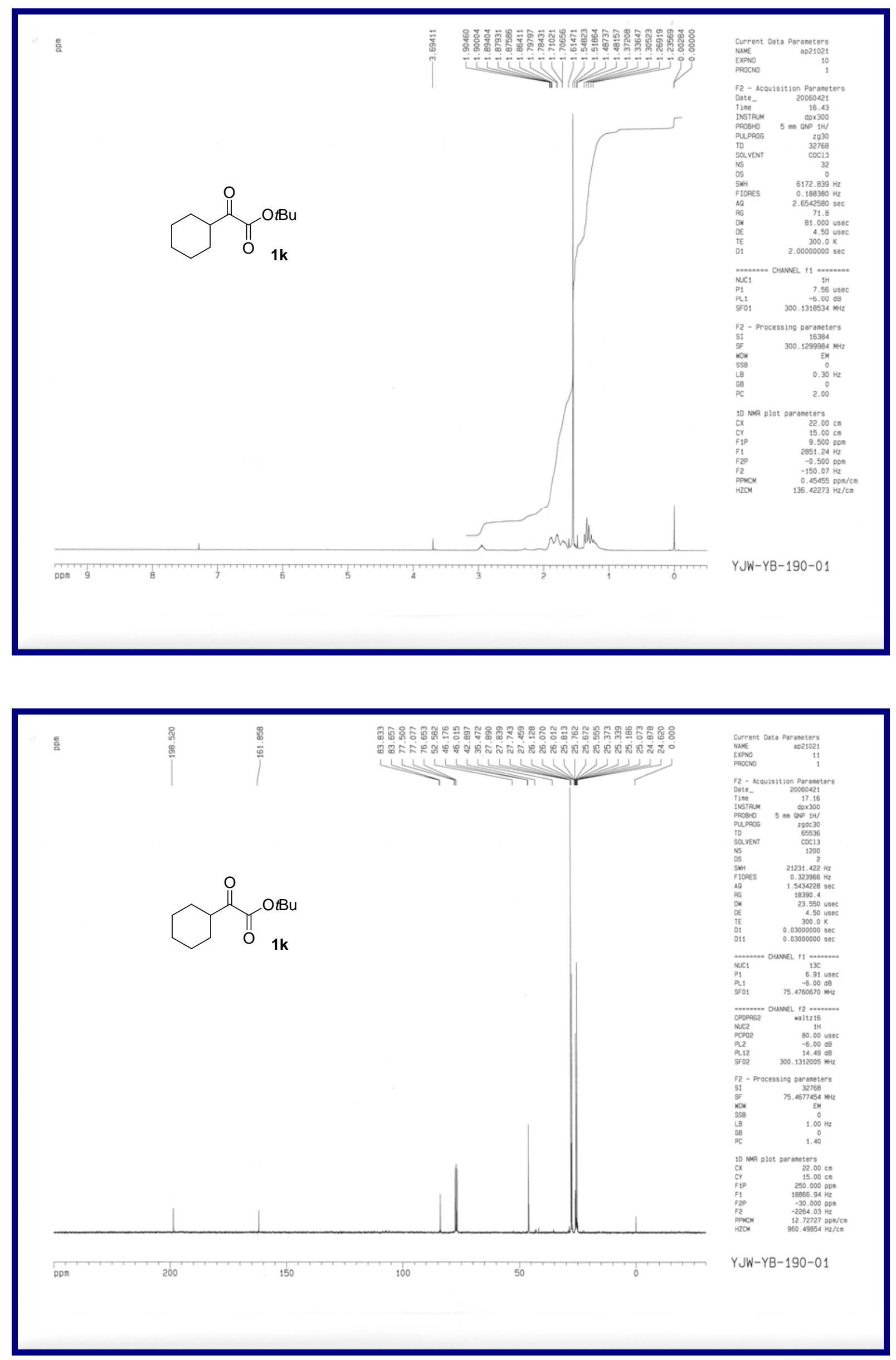

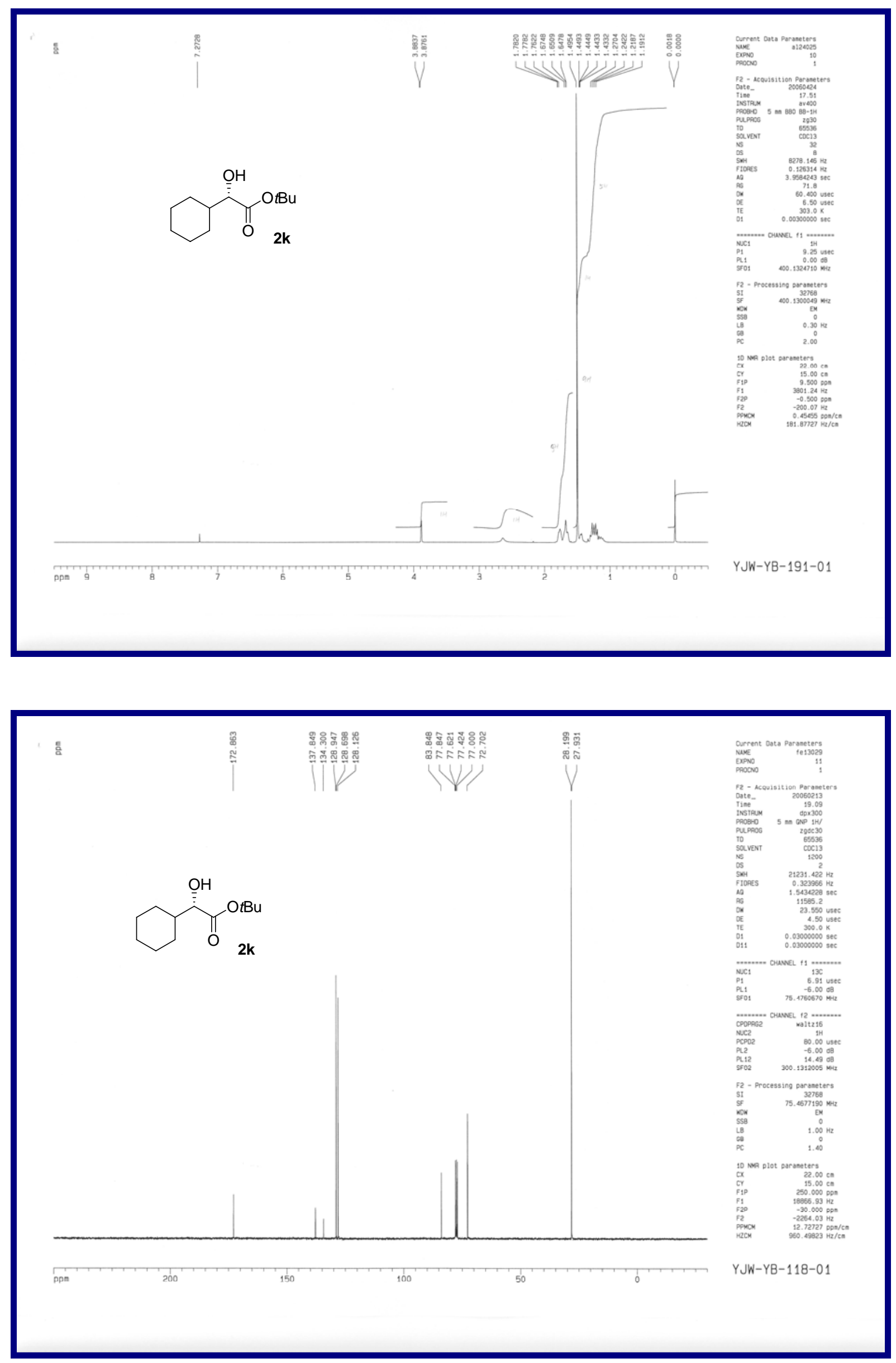

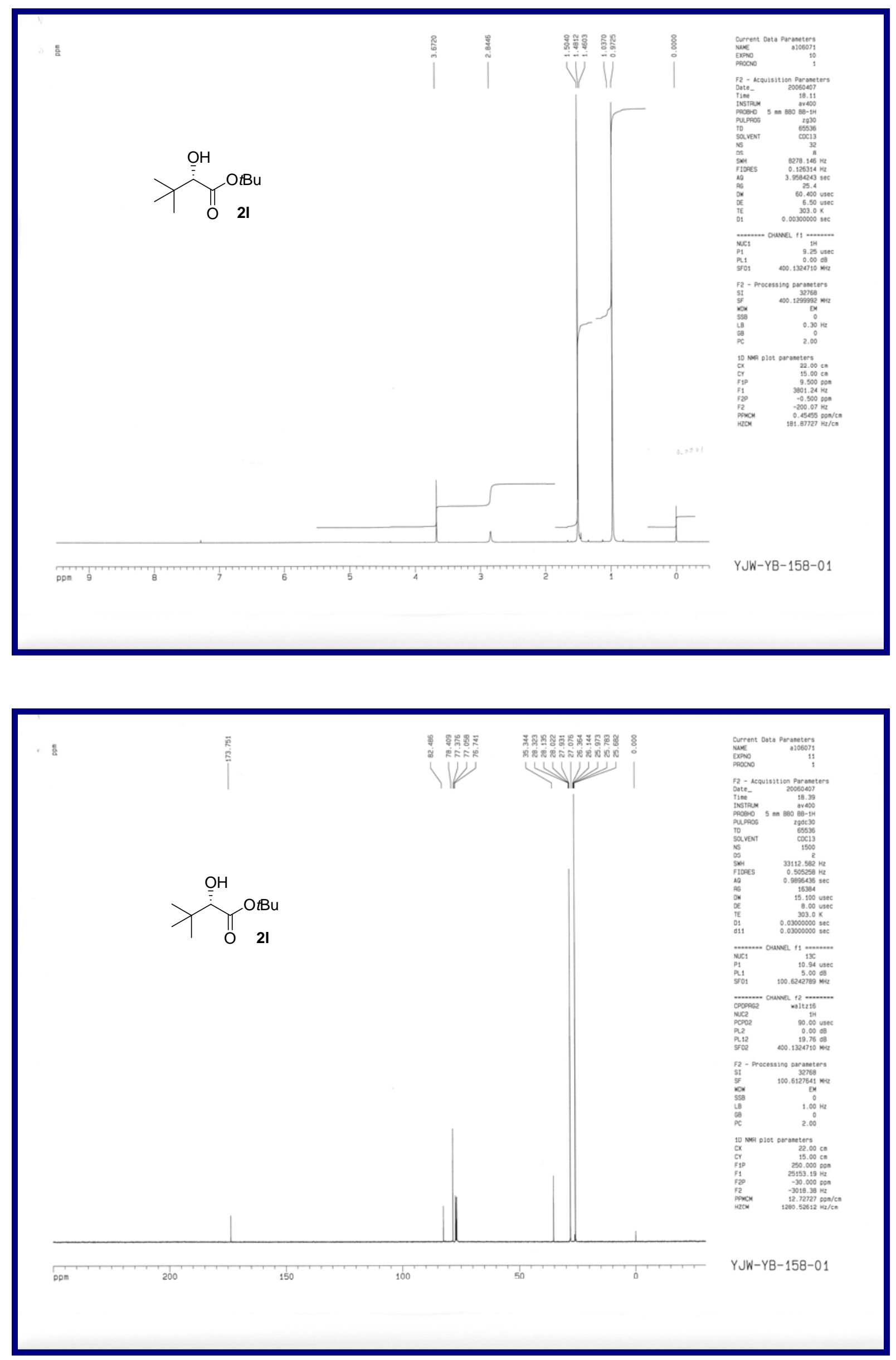

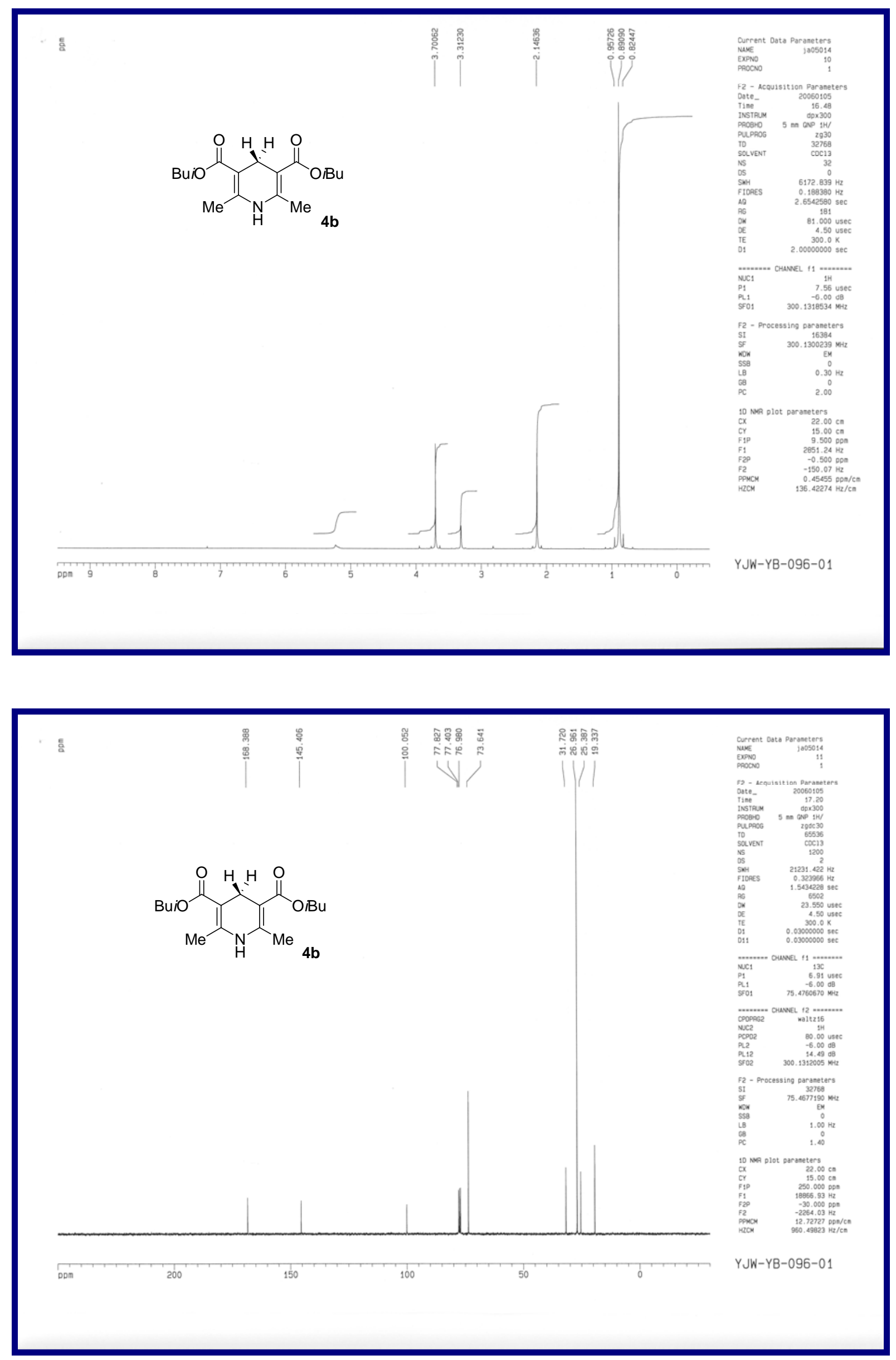